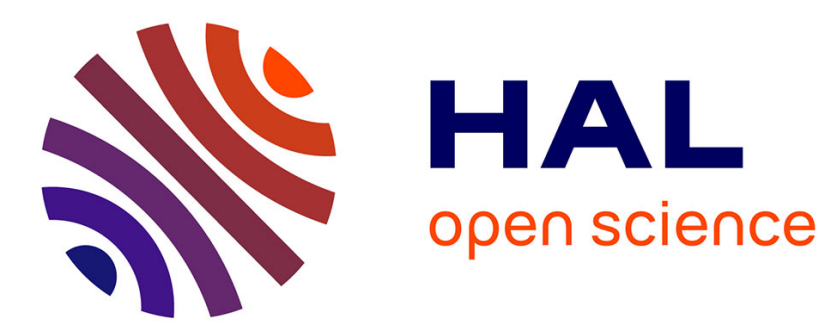

\title{
A reduced MHD model for ITG-NTM interplay
}

\author{
J Frank, O. Agullo, P. Maget, X. Garbet, N. Dubuit, M. Muraglia
}

\section{To cite this version:}

J Frank, O. Agullo, P. Maget, X. Garbet, N. Dubuit, et al.. A reduced MHD model for ITG-NTM interplay. Physics of Plasmas, 2020, 10.1063/1.5141933 . hal-02492726

\section{HAL Id: hal-02492726 \\ https://hal.science/hal-02492726}

Submitted on 27 Feb 2020

HAL is a multi-disciplinary open access archive for the deposit and dissemination of scientific research documents, whether they are published or not. The documents may come from teaching and research institutions in France or abroad, or from public or private research centers.
L'archive ouverte pluridisciplinaire HAL, est destinée au dépôt et à la diffusion de documents scientifiques de niveau recherche, publiés ou non, émanant des établissements d'enseignement et de recherche français ou étrangers, des laboratoires publics ou privés. 


\section{A reduced MHD model for ITG-NTM interplay}

J. Frank, ${ }^{1,2}$ O. Agullo, ${ }^{1}$ P. Maget, ${ }^{2}$ X. Garbet, ${ }^{2}$ N. Dubuit, ${ }^{1}$ and M. Muraglia ${ }^{1}$

1) Aix-Marseille Université, CNRS, PIIM UMR 7345, Marseille, France

${ }^{2)}$ CEA, IRFM, 13108 St-Paul-Lez-Durance, France

(Dated: 28 January 2020)

A 6-field reduced-MHD model is derived for plasma dynamics. The new model describes coherently both Ion Temperature Gradient mode and Tearing mode, and includes neoclassical effects. The model allows the construction of an energy-like quantity with a linear pressure contribution that is conserved except for dissipative, finite Larmor radius and neoclassical terms. This model may be used to study the nonlinear interaction between ITG microturbulence and neoclassical tearing mode, which is responsible for large-scale magnetic islands in tokamaks, and opens the way to a coherent description of turbulent impurity transport in magnetic islands.

\section{INTRODUCTION}

The interaction between turbulence and MHD phenomena in fusion device plasmas has received growing attention in the last decade, both in experimental ${ }^{1-10}$ and theoretical context ${ }^{11-23}$. Basically, core MHD activity is dominated by sawteeth and neoclassical tearing modes (NTMs). The latter can be driven unstable by the current or seeded nonlinearly by turbulence ${ }^{22,24,25}$, edge localized modes (ELMs) ${ }^{26}$ or internal kink mode crashes. If the radial width of the seed exceeds a critical value, neoclassical effects, notably the bootstrap current, amplify the island width further. A large island, called NTM, can then emerge. It can exceed a few percent of the tokamak minor radius. It induces a large heat transport that flattens the temperature profile inside the separatrix and therefore degrades the energy confinement of the tokamak.

Even if it has been shown that turbulence can potentially seed magnetic island using minimal MHD models, it remain unclear whether or not core turbulence, which is driven in most cases by ion temperature gradient (ITG) instabilities, can induce or ease the emergence of NTMs and/or amplify them. It remains also to quantify the impact of neoclassical physics in that context. To clarify these open questions, one must consider a model which includes the ITG mode coherently with island dynamics. Thus the model must include density evolution, ion and electron temperature evolutions, as well as magnetic flux and perpendicular flow. Moreover, as in modern tokamaks, the parallel velocity is frequently much higher than the perpendicular one and its magnitude can inhibit or favor the triggering of islands, one should also take it into consideration.

Studies of the interplay between ITG and islands have been mostly performed using a static magnetic island and are mostly focused on transport. Various authors ${ }^{11,21,27-29}$ have, indeed, studied the lowering or the suppression of turbulence inside a magnetic island, the limitation of temperature flattening inside the island due to turbulence spreading at its edge or the formation of $\mathrm{E} \times \mathrm{B}$ shear flows that suppress turbulence in the neighbourhood of an island. The use of a static island is motivated by the fact that a magnetic island is a large-scale mode that evolves very slowly compared to the small-scale ITG turbulence. The simulation of its evolution in the presence of turbulence would therefore require very long simulations with high spatial resolution. Also, considering a static island allows to use an electrostatic model

Another concern related to magnetic islands is their potential to favour the inward transport of heavy impurities like tungsten towards the core plasma. This effect has been observed experimentally ${ }^{30,31}$ and it is again attributed to the interplay of magnetic island, turbulence and neoclassical physics. Hender ${ }^{30}$ argues that the temperature flattening inside the island on the one hand increases the neoclassical inward convection of tungsten and on the other hand decreases the diffusivity due to ITG turbulence.

For a better understanding of these numerous entanglements between turbulence and magnetic islands we would like to model the evolution of a magnetic island in the presence of turbulence in one simulation. To limit the simulation expense due to the disparate spatial and temporal scales we use the simplest model possible that is able to capture the relevant physics, which is a reduced MHD model. To our knowledge, there exists no RMHD model that describes both magnetic island evolution including neoclassical effect and the ITG mode.

In this paper, we derive a new 6-field RMHD model that is designed to describe both neoclassical tearing modes and ITG modes. Two fields, the evolution of the magnetic potential $\psi$ and the electrostatic potential $\phi$, are required to describe the tearing mode. Three more fields describing the density and the electron and ion temperature evolution are then necessary for the ITG mode, as will be explained in the section dedicated to the linear stability analysis of our model. We furthermore add the parallel velocity $u_{\|}$as the sixth field to our model because of its crucial role in neoclassical physics and equilibria, as well as impurity transport.

This paper is organised as follows. In chapter 2, we explain the derivation of our 6-field RMHD model. In a first step, we present the evolution equations in their most general form. We then describe the transition to single-helicity geometry that is appropriate to describe instabilities located around a single resonant surface, and the reduction to two-dimensional slab geometry. We dedicate a separate paragraph to the choice of a closure relation for the stress tensor and the resulting neoclassical terms that are appropriate for our simplified geometry.

In chapter 3, we present some basic properties of our model. We discuss its energy conservation properties and demonstrate the existence of relevant instabilities, notably the ITG mode. We present linear simulations to characterise the ITG mode with and without magnetic shear and discuss the impact of our 
neoclassical terms on the ITG mode. A conclusion follows.

\section{MODEL EQUATIONS}

\section{A. RMHD equations in general form}

In this section we derive a set of reduced MHD equations. We consider a tokamak plasma with small inverse aspect ratio $\varepsilon_{g}=r / R$, with $R$ the major Radius of the tokamak and $r$ the minor radius of the considered resonant surface. The typical frequency $\omega$ of the phenomena of interest is supposed to be much smaller than the ion cyclotron frequency $\Omega_{i}$, so that $\varepsilon_{\omega}=\omega / \Omega_{i} \ll 1 . \varepsilon_{g}$ and $\varepsilon_{\omega}$ are used as ordering parameters We start from Braginskii's equations ${ }^{32}$.

$$
\begin{aligned}
\partial_{t} n+\nabla \cdot\left(n \mathbf{u}_{s}\right)= & 0 \\
n m_{s}\left(\partial_{t}+\mathbf{u}_{s} \cdot \nabla\right) \mathbf{u}_{s}= & e_{s} n\left(\mathbf{u}_{s} \times \mathbf{B}+\mathbf{E}\right) \\
& -\nabla p_{s}-\nabla \cdot \overline{\bar{\Pi}}_{s}+\mathbf{R}_{s} \\
\frac{3}{2} \partial_{t} p_{s}+\frac{3}{2}\left(\mathbf{u}_{s} \cdot \nabla\right) p_{s}= & -\frac{5}{2} p_{s} \nabla \cdot \mathbf{u}_{s}-\nabla \cdot \mathbf{q}_{s} \\
& -\overline{\bar{\Pi}}_{s}: \nabla \mathbf{u}_{s}+Q_{s}
\end{aligned}
$$

The subscript $s$ denotes the particle species. It is omitted for the density $n$ since we assume quasineutrality. $\mathbf{R}_{s}$ and $Q_{s}$ designate the generation of momentum and heat by collisions. In order to obtain neoclassical terms like the bootstrap current, an appropriate closure for the stress tensor $\overline{\bar{\Pi}}_{s}$ is needed. This will be discussed in section II C.

The reduced MHD equations are obtained by expressing the ion velocity $\mathbf{u}_{i}$ in terms of the parallel velocity component $u_{\|}$ along the magnetic field direction $\mathbf{b}=\mathbf{B} /|B|$ and the perpendicular drift velocities: The $\mathrm{E} \times \mathrm{B}$ drift $\mathbf{u}_{E}$, the diamagnetic drifts due to pressure and stress tensor $\mathbf{u}_{p}^{*}$ and $\mathbf{u}_{\Pi}^{*}$ and the polarisation drift velocity $\mathbf{u}_{\mathrm{pol}}{ }^{33}$. $\mathbf{u}_{\mathrm{pol}}$ is relevant only for the ion density evolution and otherwise neglected since $\mathbf{u}_{\mathrm{pol}} / \mathbf{u}_{E} \backsim \varepsilon_{\omega}$.

$$
\mathbf{u}_{i}=u_{\|} \mathbf{b}+\mathbf{u}_{E}+\mathbf{u}_{p}^{*}+\mathbf{u}_{\Pi}^{*}+\mathbf{u}_{\mathrm{pol}}
$$

We define the total plasma velocity $\mathbf{u}$ as the mass-weighted sum of the electron and ion velocities $\mathbf{u}_{e}$ and $\mathbf{u}_{i}$. The plasma current $\mathbf{J}$ is given by $\left(\mathbf{u}_{i}-\mathbf{u}_{e}\right) e n$. Because of the small mass ratio $m_{e} / m_{i}$, we use the approximations $\mathbf{u}_{i}=\mathbf{u}$ and $\mathbf{u}_{e}=\mathbf{u}-$ $\mathbf{J} /($ en $)$.

Ohm's law is obtained from the parallel momentum equation (2) for the electron species. Due to the small electron mass the left hand side term can be neglected. We then take the projection on the parallel direction. The parallel component of $\mathbf{R}_{e}$ is given by Braginskii ${ }^{32}$ as $R_{\| e}=e^{-1} m_{e} v_{e i} J_{\|}$ $0.71 n \nabla_{\|} T_{e} \approx \eta e n J_{\|}$We have neglected the parallel therma force and introduced the Spitzer resistivity $\eta=m_{e} v_{e i} /\left(n e^{2}\right)$. These operations result in:

$$
\partial_{t} \psi=\nabla_{\|} \phi-\frac{\nabla_{\|} p_{e}}{e n_{0}}-\frac{\mathbf{b} \cdot\left(\nabla \cdot \overline{\bar{\Pi}}_{e}\right)}{e n_{0}}+\eta \tilde{J}_{\|}
$$

The evolution of the total plasma flow $\mathbf{u}$ is described by the evolution of the parallel velocity component $u_{\|}$and the vorticity $\omega$ defined below. Their evolution equations are derived by taking the sum of the momentum equations (2) for electrons and ions. The momentum exchange terms cancel because $\mathbf{R}_{i}=-\mathbf{R}_{e}$. The electron's contribution to the stress tensor $\overline{\bar{\Pi}}_{e}$ is order of $\sqrt{m_{e} / m_{i}}$ smaller than the ion's contribution and therefore neglected. In the momentum term on the left hand side the diamagnetic drift due to the stress tensor and the polarisation drift are neglected. By applying the gyroviscous cancellation eq. (34) as computed by Smolyakov ${ }^{33}$, we eliminate the flow advection term by the diamagnetic drift $\mathbf{u}_{p}^{*}$. We obtain the intermediate result:

$$
\begin{aligned}
& n m_{i}\left(\partial_{t}+\left(u_{\|} \mathbf{b}+\mathbf{u}_{E \times B}\right) \cdot \nabla\right)\left(u_{\|} \mathbf{b}+\mathbf{u}_{E \times B}+\mathbf{u}_{p_{i}}^{*}\right) \\
& =\mathbf{J} \times \mathbf{B}-\nabla\left(p_{e}+p_{i}\right)-\nabla \cdot \overline{\bar{\Pi}}_{i}
\end{aligned}
$$

We project this equation on the parallel direction by multiplying with $\mathbf{b}$. We simplify the expression by neglecting terms of higher order in $\varepsilon$, notably curvature terms, and cubic terms. Consequently, we replace the density by the mean density $n_{0}$. Using the Poisson bracket notation $\mathbf{b} \cdot(\nabla f \times \nabla g)=\{f, g\}$, we obtain the evolution equation for the parallel velocity:

$$
\partial_{t} u_{\|}=-\frac{1}{B}\left\{\phi, u_{\|}\right\}-\frac{\nabla_{\|}\left(p_{i}+p_{e}\right)}{m_{i} n_{0}}-\frac{\mathbf{b} \cdot\left(\nabla \cdot \overline{\bar{\Pi}}_{i}\right)}{m_{i} n_{0}}
$$

We recast the perpendicular projection of equation (6) into a vorticity evolution equation by taking the parallel (and dominant) component of the curl. We obtain a term containing the perpendicular current $\mathbf{J}_{\perp}$ which we re-express by evaluating equation (6) neglecting the higher order inertial contribution. The details of the calculation are given in appendix 1 . With the definition of the generalised vorticity $\omega=\Delta_{\perp}\left(\phi+p_{i} /\left(e n_{0}\right)\right)$ we obtain the following evolution equation:

$$
\begin{aligned}
\partial_{t} \omega= & -\frac{1}{B}\{\phi, \omega\}+B^{2} \frac{\nabla_{\|} J_{\|}}{n_{0} m_{i}}-\frac{2}{n_{0} m_{i}}\left\{p_{i}+p_{e}, B_{e q}\right\} \\
& +\frac{\mu}{n_{0} m_{i}} \Delta_{\perp} \tilde{\omega}-2 \frac{\nabla B}{B m_{i} n_{0}} \cdot\left(\mathbf{B} \times \nabla \cdot \overline{\bar{\Pi}}_{i}\right) \\
& -\frac{\mathbf{B}}{m_{i} n_{0}} \cdot\left(\nabla \times \nabla \cdot \overline{\bar{\Pi}}_{i}\right)
\end{aligned}
$$

The magnitude of the equilibrium magnetic field is given to leading order in $\varepsilon_{g}$ by $B_{e q}=B_{0}(1-r / R \cos (\theta))$. We now evaluate the continuity equation (1) for ions, retaining all the drift velocities in (4). Still keeping the dominant terms in the orderings, we obtain the evolution equation for the generalised ion density:

$$
\begin{aligned}
& \partial_{t}\left(n-\frac{n_{0} m_{i}}{e B_{0}^{2}} \omega\right)=-n_{0} \nabla_{\|} u_{\|}+\frac{2 n_{0}}{B_{0}^{2}}\left\{\phi, B_{e q}\right\} \\
& +\frac{1}{B_{0}}\{n, \phi\}+\frac{2}{e B_{0}^{2}}\left\{p_{i}, B_{e q}\right\} \\
& +\frac{n_{0} m_{i}}{e B_{0}^{3}}\{\phi, \omega\}-\nabla \cdot \frac{\mathbf{B} \times \nabla \overline{\bar{\Pi}}_{i}}{e B^{2}}
\end{aligned}
$$

Combining (8) and (9) gives the evolution equation for the density we would obtain directly from the electron continuity equation. This comes from the fact that quasineutrality was 
already assumed in the momentum equation. Note that the neoclassical terms containing the stress tensor cancel out to leading order in $\varepsilon_{g}$. Adding a diffusive term to account for the small scale ETG turbulence, we end up with:

$$
\begin{aligned}
\partial_{t} n= & -n_{0} \nabla_{\|} u_{\|}+\frac{2 n_{0}}{B_{0}^{2}}\left\{\phi, B_{e q}\right\}+\frac{1}{B_{0}}\{n, \phi\} \\
& -\frac{2}{e B_{0}^{2}}\left\{p_{e}, B_{e q}\right\}+\frac{1}{e} \nabla_{\|} J_{\|}+D \Delta_{\perp} \tilde{n}
\end{aligned}
$$

In the remainder of the paper, we will use the electron density equation (10) for simplicity.

Finally, we obtain the evolution equations for the electron and ion pressure from the energy balance equation (3). We will assume that the heat source term is balanced by equilibrium quantities

The heat fluxes are split into their parallel and perpendicular contributions following Braginskii ${ }^{32}$, with $q_{\| s}=-\chi_{\| s} \nabla_{\|} T_{s}$ and $q_{\perp s}=-\chi_{\perp s} \nabla_{\perp} T_{s}$. The following gyroviscous cancellation is derived in appendix 2 :

$\frac{3}{2} \mathbf{u}_{p_{s}}^{*} \cdot \nabla p_{s}+\frac{5}{2} p_{s} \nabla \cdot \mathbf{u}_{p_{s}}^{*}+\nabla \cdot \mathbf{q}_{\wedge s}=\frac{5}{2} \nabla \cdot\left(\frac{\mathbf{B}}{e_{s} B^{2}} \times \nabla\left(p_{s} T_{s}\right)\right)$

Considering $\varepsilon_{\omega} \ll \varepsilon_{g}$ and that advective terms are of order $\varepsilon_{\omega}$ and divergence terms linked to curvature are of order $\varepsilon_{g}$, we retain the diamagnetic drift due to the stress tensor only in the divergence term:

$$
\begin{aligned}
& \frac{3}{2}\left(\partial_{t}+\left(\mathbf{u}_{\| s}+\mathbf{u}_{E \times B}+\mathbf{u}_{s \nabla p}\right) \cdot \nabla\right) p_{s} \\
= & -\frac{5}{2} p_{s} \nabla \cdot\left(\mathbf{u}_{s \|}+\mathbf{u}_{E \times B}+\mathbf{u}_{s \nabla p}+\mathbf{u}_{s \nabla \Pi}\right) \\
& -\nabla_{\|} q_{s \|}-\nabla \cdot \mathbf{q}_{\perp s}-\nabla \cdot \mathbf{q}_{\wedge s}
\end{aligned}
$$

After elimination of higher-order terms the ion pressure evolution equation becomes:

$$
\begin{aligned}
& \partial_{t} p_{i}=-\frac{1}{B}\left\{\phi, p_{i}\right\}+\frac{10}{3} \frac{p_{i}}{B^{2}}\left\{\phi, B_{e q}\right\}+\frac{10}{3} \frac{T_{i}}{e B^{2}}\left\{p_{i}, B_{e q}\right\} \\
& +\frac{10}{3} \frac{p_{i}}{e B^{2}}\left\{T_{i}, B_{e q}\right\}-\frac{5}{3} p_{i} \nabla_{\|} u_{\|}+\frac{2}{3} \chi_{\perp i} \Delta_{\perp} T_{i}+\frac{2}{3} \chi_{\| i} \Delta_{\|} T_{i} \\
& +\frac{5}{3} \frac{p_{i}}{e n} \frac{\nabla B}{B} \cdot\left(\frac{2 \mathbf{B}}{B^{2}} \times\left(\nabla \cdot \overline{\bar{\Pi}}_{i}\right)\right)+\frac{5}{3} p_{i} \frac{\mathbf{B}}{B^{2}} \cdot\left(\nabla \times \frac{\nabla \cdot \overline{\bar{\Pi}}_{i}}{e n}\right)(12)
\end{aligned}
$$

The electron pressure equation takes a similar form. Main differences are the parallel current appearing due to $u_{\| e}=u_{\|}$ $J_{\|} /(e n)$ and the neglect of the stress tensor terms because of the small electron mass.

\section{B. Reduction to 2D single-helicity geometry}

It is convenient to focus on the dynamics in the vicinity of a low order resonant surface where potentially NTMs grow. The magnetic field expression reads:

$$
\mathbf{B}=\mathbf{B}_{r e s}+\nabla \psi \times \nabla \varphi
$$

Here $\mathbf{B}_{r e s}$ is the equilibrium magnetic field at the resonance, which is in toroidal coordinates $r, \theta, \varphi$ given by $\mathbf{B}_{r e s}=$
$B_{0}\left(\mathbf{e}_{\varphi}+r /\left(q_{\text {res }} R\right) \mathbf{e}_{\theta}\right)$, with $q_{\text {res }}$ the safety factor at resonance. The magnetic flux variable $\psi$ is used to describe both the radial shear of the equilibrium magnetic field and fluctuations of the magnetic field. The change of variables

$$
\begin{aligned}
r^{\prime} & =r \\
\theta^{\prime} & =\theta-\frac{\varphi}{q_{\text {res }}} \\
\varphi^{\prime} & =\varphi
\end{aligned}
$$

allows us to express the parallel derivative simply as $\nabla_{\|} f=\mathbf{b}$. $\nabla f=\frac{1}{R} \partial_{\varphi^{\prime}}+\frac{1}{R B_{0}}\{\psi, f\}_{r^{\prime} \theta^{\prime}}$. Single helicity hypothesis means fields are functions of $r^{\prime}$ and $\theta^{\prime}$ only.

As a further simplification we can use the slab approximation, which means that we consider a plasma volume at low field side $\theta=0$ that is small enough to replace the curvilinear coordinates $r^{\prime}, \theta^{\prime}$ with Cartesian coordinates $x, y$. In this step, the poloidal variations of the equilibrium magnetic field are lost.

Note that this last simplification cannot be used for the study of effects that depend on the poloidal variation of the equilibrium magnetic field, for example neoclassical transport of impurities.

\section{Closure for the stress tensor}

We need to introduce a closure relation for the stress tensor $\bar{\Pi}_{s}$, which accounts for neoclassical physics in our equation set. Closure relations that are commonly used in numerical codes are designed to reproduce the flux surface averaged quantities predicted by neoclassical theory. Various heuristic closures are proposed for example by Gianakon ${ }^{34}$. Here we use equation (12) therein which gives an easy expression for the viscous force. A similar expression was derived earlier in $^{35}$.

$$
\nabla \cdot \overline{\bar{\Pi}}_{s}=m_{s} n_{0} \mu_{s}^{n c}\left\langle B^{2}\right\rangle \frac{\mathbf{v}_{s} \cdot \hat{\mathbf{e}}_{\theta}}{B_{\theta}^{2}} \hat{\mathbf{e}}_{\theta}
$$

In order to recover the correct form of the surface averaged viscous force $\left\langle\mathbf{B} \cdot \nabla \cdot \overline{\bar{\Pi}}_{s}\right\rangle$ as given by Hirshmann ${ }^{36}$, eq. (4.18), we identify $\mathbf{v}_{s}=\mathbf{u}_{s}+\frac{2}{5} k_{s} \frac{\mathbf{q}_{s}}{p_{s}}$ as the sum of the velocity $\mathbf{u}_{s}=u_{\| s} \mathbf{b}+\mathbf{u}_{E \times B}+\mathbf{u}_{p_{i}}^{*}$ and the heat flux $\mathbf{q}_{\theta s}=q_{\| s} \mathbf{b}+\mathbf{q}_{\triangle s}$. The parameter $k_{s}=\mu_{s 2} / \mu_{s 1}$ designates the ratio of the viscosity coefficients $\mu_{s 1,2}$. The viscous damping frequency $\mu_{s}^{n c}$ is given by ${ }^{34}$ as a function of the collision frequency, $\varepsilon_{g}$ and the trapped particle fraction, but for simplicity we use $\mu_{s}^{n c}=\sqrt{\varepsilon_{g}} v_{s}$.

We use the closure (15) to compute the neoclassical terms in equations (5),(7), (8) and (12). We average the resulting expressions over the poloidal direction to eliminate the $\cos (\theta)$ dependence of the equilibrium magnetic field, assuming that poloidal variations of all other quantities are negligible. We also assume that the averaged parallel heat flux $\left\langle B q_{\| s}\right\rangle$ is zero, so that this contribution to $\mathbf{v}_{s}$ vanishes. Some models ${ }^{37-39}$ that use this closure to obtain averaged neoclassical terms neglect the heat flux contribution altogether, setting $\mathbf{v}_{s}=\mathbf{u}_{s}$ 
If we want to avoid the slab approximation in our model and wish to retain the $\theta$ dependence of the magnetic field even in the neoclassical terms, a more sophisticated closure would be more appropriate. This alternative closure is based on the $\mathrm{CGL}^{40}$ formulation for the stress tensor:

$$
\overline{\bar{\Pi}}=\frac{3}{2} \pi_{\|}\left(\mathbf{b b}-\frac{1}{3} \mathbb{I}\right) \quad \text { with } \quad \pi_{\|}=\frac{2}{3}\left(p_{\|}-p_{\perp}\right)
$$

Here $p_{\|}$and $p_{\perp}$ designate the pressure parallel and perpedicular to the magnetic field, respectively. Together with the closure relation for the pressure anisotropy

$$
\frac{3}{2} \pi_{\| s}=-\frac{n m_{s} \mu_{s} B^{2}}{\left\langle(\mathbf{b} \cdot \nabla B)^{2}\right\rangle} \mathbf{v}_{s} \cdot \frac{\nabla B}{B}
$$

we obtain neoclassical terms that are, after poloidal averaging, equivalent to the results obtained from equation (15). The details of the calculation of the neoclassical terms for our model as well as a comparison between the two closures are given in appendix 3 .

\section{Normalisation and summary}

We normalize temporal and spatial scales to the Alfvén time $\tau_{A}=L_{\perp} / v_{A}$ and a typical perpendicular scale length $L_{\perp}$ of our system. Density and magnetic field are normalised to their values at the resonance $n_{0}$ and $B_{0}$. The parallel velocity $u_{\|}$is normalised to the Alfvén velocity $v_{A}$. The normalisation of the pressure and electrostatic potential is chosen such that the $\mathrm{E} \times \mathrm{B}$ and diamagnetic drift velocities are given in units of the Alfvén velocity, that is $\phi=L_{\perp} B_{0} v_{A} \phi_{N}$ and $p_{s}=p_{s N} p_{0} / p_{0 N}$ with $p_{0 N}=\beta_{0} /\left(2 \Omega_{i} \tau_{A}\right)$ where $p_{0}$ and $\beta_{0}$ are the pressure and plasma beta value at resonance and $\Omega_{i}$ the ion cyclotron frequency. We introduce several dimensionless parameters for notation. The equilibrium pressure gradient $v^{*}=p_{0 N} L_{\perp} / L_{p}$ is given by the pressure scale length $L_{p}$. The two curvature parameters $\kappa_{1}=2 \Omega_{i} \tau_{A} L_{\perp} / R_{0}$ and $\kappa_{2}=-10 / 3 \cdot L_{p} / R_{0}$ are related through $\kappa_{2}=-\kappa_{1} \rho_{*}^{2} / v^{*}$; however we want to be able to change them independently as a tool to tweak the linear spectrum, in order to compensate for our $2 \mathrm{D}$ geometry. Finally we define the normalised Larmor radius as $\rho_{*}^{2}=5 / 3 \cdot p_{0 N} /\left(\tau_{A} \Omega_{i}\right)$. Our six equations derived above, after the transition to single-helicity 2D slab geometry, then become :

$$
\begin{aligned}
\partial_{t} \psi= & \{\psi, \phi\}-\left\{\psi, p_{e}\right\}+\eta^{n c}\left(\tilde{J}_{\|}-\tilde{J}_{b s}\right) \\
\partial_{t} \omega= & -\{\phi, \omega\}+\left\{\psi, J_{\|}\right\}-\kappa_{1} \partial_{y}\left(p_{i}+p_{e}\right)+\mu \Delta_{\perp} \tilde{\omega} \\
& -\mu_{i}^{n c}\left(\frac{q}{\varepsilon_{g}}\right)^{2} \partial_{x}\left(u_{\theta}+k_{i} \partial_{x} T_{i}\right) \\
\partial_{t} n= & -\left\{\psi, u_{\|}\right\}+\frac{1}{\Omega_{i} \tau_{A}}\left\{\psi, J_{\|}\right\}+\{n, \phi\} \\
& +\frac{3}{5} \frac{\kappa_{2} v^{*}}{p_{0 N}} \partial_{y}\left(p_{e}-\phi\right)+D \Delta_{\perp} \tilde{n} \\
\partial_{t} p_{i}= & -\left\{\phi, p_{i}\right\}-\frac{5}{3} p_{i}\left\{\psi, u_{\|}\right\}-\frac{\kappa_{2} v^{*}}{p_{0 N}} p_{i} \partial_{y} \phi \\
& -\frac{\kappa_{2} v^{*}}{p_{0 N}} \partial_{y}\left(T_{i} p_{i}\right)+\chi_{\perp i} \Delta_{\perp} \tilde{T}_{i}+\chi_{\| i}\left\{\psi,\left\{\psi, \tilde{T}_{i}\right\}\right\} \\
& +\mu_{i}^{n c} \rho_{*}^{2} \frac{\tau_{i}}{1+\tau_{i}}\left(\frac{q}{\varepsilon_{g}}\right)^{2} \partial_{x}\left(u_{\theta}+k_{i} \partial_{x} T_{i}\right) \\
\partial_{t} p_{e}= & -\left\{\phi, p_{e}\right\}+\frac{5}{3} \frac{1}{\Omega_{i} \tau_{A}} p_{e}\left\{\psi, J_{\|}\right\}-\frac{5}{3} p_{e}\left\{\psi, u_{\|}\right\} \\
& -\frac{\kappa_{2} v^{*}}{p_{0 N}} p_{e} \partial_{y} \phi+\frac{\kappa_{2} v^{*}}{p_{0 N}} \partial_{y}\left(T_{e} p_{e}\right) \\
& +\chi_{\perp e} \Delta_{\perp} \tilde{T}_{e}+\chi_{\| e}\left\{\psi,\left\{\psi, \tilde{T}_{e}\right\}\right\} \\
\partial_{t} u_{\|}= & -\left\{\phi, u_{\|}\right\}-\Omega_{i} \tau_{A}\left\{\psi, p_{i}+p_{e}\right\} \\
& -\mu_{i}^{n c} \frac{q}{\varepsilon_{g}}\left(u_{\theta}+k_{i} \partial_{x} T_{i}\right)
\end{aligned}
$$

We have used the following definitions:

$$
\begin{aligned}
\omega & =\Delta_{\perp}\left(\phi+p_{i}\right) \\
J_{\|} & =\Delta_{\perp} \psi \\
\eta^{n c} & =\eta\left(1+\frac{\mu_{e}}{v_{e}}\right) \\
J_{b s} & =\frac{\mu_{e}}{v_{e i}+\mu_{e}} \Omega_{i} \tau_{A}\left(u_{\|}+\frac{q}{\varepsilon_{g}} \partial_{x}\left(\phi-p_{e}-k_{e} T_{e}\right)\right) \\
u_{\theta} & =\frac{\varepsilon_{g}}{q} u_{\|}+\partial_{x}\left(\phi+p_{i}\right) \\
\tau_{i} & =T_{e, e q} / T_{i, e q}
\end{aligned}
$$

All fields include equilibrium and fluctuating part, e.g. $\psi(x, y)=\psi_{e q}(x)+\tilde{\psi}(x, y)$, except if a field is marked explicitly by a $\approx$ as a fluctuation. As long as our equilibrium fields depend only on the radial direction $x$, we may choose them freely, as long as we respect the condition $u_{\theta, e q}=-k_{i} \partial_{x} T_{i, e q}$ imposed by the neoclassical terms.

\section{MODEL PROPERTIES}

\section{A. Engergy balance}

When deriving a set of reduced MHD equations, the perfect energy conservation inherent to Braginskii's equations is lost. Often energy-like quantities that are conserved except for inherently dissipative terms can be constructed. The pressure 
contribution of these energies is often quadratic, as in the case of Hazeltine's four-field mode ${ }^{41}$ or in 3-field models ${ }^{42}$. In our model, we chose to keep certain cubic terms in the pressure equation that allow for a linear pressure energy. For the sake of clarity we consider the energy contributions from the electrons $E_{\text {elec }}$, the ions $E_{\text {ions }}$ and from the electromagnetic field $E_{\text {field }}$ separately:

$$
\begin{aligned}
E_{\text {total }} & =E_{\text {elec }}+E_{\text {ions }}+E_{\text {field }} \\
E_{\text {elec }} & =\int \mathrm{d}^{3} x\left(\frac{3}{5} \Omega_{i} \tau_{A} p_{e}\right) \\
E_{\text {ions }} & =\int \mathrm{d}^{3} x\left(\frac{3}{5} \Omega_{i} \tau_{A} p_{i}+\frac{\left|u_{\|}\right|^{2}}{2}\right) \\
E_{\text {field }} & =\int \mathrm{d}^{3} x\left(\frac{\left|\nabla_{\perp} \psi\right|^{2}}{2}+\frac{\left|\nabla_{\perp}\left(\phi+p_{i}\right)\right|^{2}}{2}\right)
\end{aligned}
$$

The variations of these energy contributions are readily obtained from the evolution equations (18) to (23) by partial integration. For all our fields $\tilde{f}$ we use periodic boundary conditions in $y$-direction and Dirichlet boundary conditions $\tilde{f}=0$ in the $x$-direction. Additionally we choose $\psi_{e q}(x)=0$ and $\omega_{e q}(x)=0$ at the boundaries. Under these conditions the boundary flux terms vanish and we find for example for the electron species:

$$
\begin{aligned}
\partial_{t} E_{\text {elec }} & =\delta E_{i \rightarrow e}+\delta E_{f \rightarrow e}+\delta_{t} E_{\text {diss }, e} \\
\delta E_{i \rightarrow e} & =\int \mathrm{d}^{3} x\left(-\Omega_{i} \tau_{A} p_{e}\left\{\psi, u_{\|}\right\}\right) \\
\delta E_{f \rightarrow e} & =\int \mathrm{d}^{3} x\left(p_{e}\left\{\psi, J_{\|}\right\}+\frac{\kappa_{2} v^{*}}{\rho_{*}^{2}} \phi \partial_{y} p_{e}\right) \\
\delta_{t} E_{\text {diss }, e} & =\int \mathrm{d}^{3} x\left(\frac{3}{5} \Omega_{i} \tau_{i} \chi_{\perp e} \Delta_{\perp} \tilde{T}_{e}\right)
\end{aligned}
$$

Here $\delta E_{i \rightarrow e}$ designates the energy transfer from the ions to the electrons and $\delta E_{f \rightarrow e}$ is the energy transfer from the field to the electrons. $\delta E_{\text {diss, }}$ is the dissipative term related to the electron temperature fluctuations. For the variation of $E_{\text {field }}$ we obtain additionally a number of unbalanced terms $\delta E_{\text {Larmor }}$ that are related to finite Larmor radius effects:

$$
\begin{aligned}
\partial_{t} E_{\text {field }}= & \delta E_{e \rightarrow f}+\delta E_{i \rightarrow f}+\delta E_{\text {diss }}+\delta E_{\text {Larmor }} \\
\delta E_{e \rightarrow f}= & \int \mathrm{d}^{3} x\left(J_{\|}\left\{\psi, p_{e}\right\}+\kappa_{1} \phi \partial_{y} p_{e}\right) \\
\delta E_{i \rightarrow f}= & \int \mathrm{d}^{3} x \kappa_{1} \phi \partial_{y} p_{i} \\
\delta E_{\text {diss }}= & \int \mathrm{d}^{3} x\left(-\eta J_{\|} \tilde{J}_{\|}-\mu \omega \tilde{\omega}+\ldots\right. \\
& \left.\ldots+\frac{3}{5} \Omega_{i} \tau_{i} \Delta_{\perp}\left(\chi_{\perp i} \tilde{T}_{i}+\chi_{\perp e} \tilde{T}_{e}\right)\right) \\
\delta E_{\text {Larmor }}= & \int \mathrm{d}^{3} x\left(p_{i}\{\phi, \omega\}-p_{i}\left\{\psi, J_{\|}\right\}+\kappa_{1} p_{i} \partial_{y} p_{e}\right)
\end{aligned}
$$

As long as the natural relation between the curvatures $\kappa_{2}=$ $-\kappa_{1} \rho_{*}^{2} / v^{*}$ is respected, the energy transfer terms between fields are perfectly balanced, e.g. $\delta E_{e \rightarrow f}=-\delta E_{f \rightarrow e}$. The variation of the total energy is therefore given by the dissipative and finite Larmor radius terms:

$$
\partial_{t} E_{\text {total }}=\delta E_{\text {diss }}+\delta E_{\text {Larmor }}
$$

In fact the finite Larmor radius contribution to the generalised vorticity $\omega=\Delta_{\perp}\left(\phi-p_{i}\right)$ is always a challenge for the energy balance. This problem can be solved by increasing the complexity of the model and introducing an equation on the perpendicular current ${ }^{43}$, or avoided by neglecting the ion pressure or at least taking the limit of vanishing Larmor radius ${ }^{41}$ $\left(\Omega_{i} \tau_{A}\right)^{-1} \rightarrow 0$ which allows to neglect $p_{i}$ in the expression of $\omega$ (recall the renormalisation of the pressure). In this limit we obtain $\delta E_{\text {Larmor }}=0$.

In this energy balance we have not considered the neoclassical terms, since they are small and result in lengthy expressions. However $\mathrm{in}^{38}$ all neoclassical contributions to the energy balance combine into purely dissipative terms. This is achieved by omitting the heat flux contribution to the stress tensor and by retaining several terms of order $\sqrt{m_{e} / m_{i}}$ that we have neglected.

\section{B. Linear analysis: Evidence of ITG mode}

We designed our model in order to describe the tearing mode, the interchange mode and the ion temperature gradient mode. The former two instabilities were already described in the former 3 -field code version ${ }^{44}$. The equations therein correspond to our equations (18), (19) and (22) with the remaining three fields $n, p_{i}$ and $u_{\|}$assumed constant and without neoclassical terms. The ITG mode is new to our model, therefore we need to verify its presence. We can reduce our 6field model to obtain a simple 2-field model that describes the electrostatic toroidal ITG mode by using only the ion pressure equation (21) and the ion density equation (9), and setting the fields $\psi, p_{e}$ and $u_{\|}$to zero. Because of the missing vorticity equation we cannot use the electron density equation (20), since we would then miss the ion inertia which is essential for the ITG mode. For an analytical treatment we consider again the limit of small Larmor radius, $\left(\Omega_{i} \tau_{A}\right)^{-1} \rightarrow 0$, and neglect dissipations:

$$
\begin{aligned}
& \partial_{t} n=\{n, \phi\}-\frac{3}{5} \frac{\kappa_{2} v^{*}}{p_{0 N}} \partial_{y}\left(p_{i}+\phi\right) \\
& \partial_{t} p_{i}=-\left\{\phi, p_{i}\right\}-\frac{\kappa_{2} v^{*}}{p_{0 N}} p_{i} \partial_{y} \phi-\frac{\kappa_{2} v^{*}}{p_{0 N}} \partial_{y}\left(T_{i} p_{i}\right)
\end{aligned}
$$

The system is closed by assuming adiabatic electron response $\tilde{\phi} p_{0 N} /\left(1+\tau_{i}\right)=\tilde{n}$. Here $\tau_{i}=T_{i} / T_{e}$ is the ion to electron temperature ratio, thus $p_{0 N} /\left(1+\tau_{i}\right)$ represents the equilibrium electron pressure. This model is similar to that of Ref. ${ }^{45}$, without trapped electrons. After linearisation of this system we obtain a dispersion relation for the toroidal ITG mode:

$$
\begin{aligned}
\omega= & k_{y} \frac{\tau_{i}}{1+\tau_{i}}\left(\frac{1}{2 \tau_{i}}\left(p_{0 N} \partial_{x} N_{0}-\frac{5}{3} \kappa_{2} v^{*}\right)-\kappa_{2} v^{*}\right) \\
& \pm k_{y} \frac{\tau_{i}}{1+\tau_{i}} \sqrt{p_{0 N} \partial_{x} N_{0} \frac{3}{5} \kappa_{2} v^{*} \frac{1}{\tau_{i}}} \sqrt{\eta_{c r}-\eta_{i}} \\
\eta_{c r}= & \frac{2}{3}-\frac{1}{2 \tau_{i}}+\frac{1}{4 \tau_{i}} \frac{3}{5} \frac{p_{0 N} \partial_{x} N_{0}}{\kappa_{2} v^{*}} \\
& +\frac{5}{3} \frac{\kappa_{2} v^{*}}{3 p_{0 N} \partial_{x} N_{0}}\left(\frac{1}{4 \tau_{i}}+\frac{10}{9} \tau_{i}\right)
\end{aligned}
$$


pictures/WEST5_Fourier_ky0p4.pdf

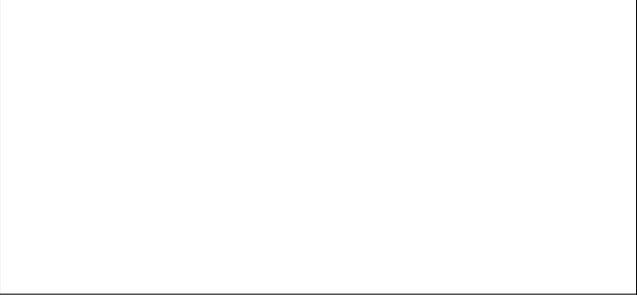

FIG. 1: Growth rates of the most unstable mode at $k_{y} \rho_{i}=0.4$ in dependence of the equilibrium density and temperature gradients. The results are obtained from Fourier analysis of our linearised 6-field model in the limit of high parallel electron heat flux and zero parallel ion heat flux, neglecting magnetic shear. The parameters are based on WEST discharge \#53259.

The parameter $\eta_{i}$ is defined as $\eta_{i}=\partial \ln \left(T_{i, e q}\right) / \partial \ln \left(n_{e q}\right)=$ $\partial_{x} p_{i, e q} /\left(p_{i, e q} \partial_{x} n_{e q}\right)-1$, where $\partial_{x} p_{i, e q}$ and $\partial_{x} n_{e q}$ are the radial gradients of the equilibrium ion pressure and density profiles, respectively. The ITG mode is unstable if $\eta_{i}$ exceeds the critical value $\eta_{c r}$. The same result was found in ${ }^{46}$, equation (5). To see that the results are identical, identify $\omega_{D}=$ $-k_{y}(3 / 5) \kappa_{2} v^{*} \tau_{i} /\left(1+\tau_{i}\right)$ and $\omega_{* i}=-k_{y} p_{0 N} \partial_{x} N_{0} \tau_{i} /\left(1+\tau_{i}\right)$ Also note the different definition of the temperature ratio $\tau$. A comparison with other models taking into account many other effects (for instance Ref. ${ }^{47}$ ) is out of the scope of this paper, since in this section we focus on recovering the fundamental traits of ITG instability.

In our 6-field model not only the toroidal ITG but also the slab ITG mode is present, due to the parallel velocity dynamics equation (23). In both cases, the adiabaticity condition is essential for the underlying instability mechanism. In our 6field model, adiabaticity is only ensured if the parallel electron heat flux is sufficiently large so that $\nabla_{\|} T_{e} \approx 0$. Together with the condition $\nabla_{\|} \phi \approx \nabla_{\|} p_{e}$ from Ohm's law equation (18), this results in $\nabla_{\|} \phi \approx \nabla_{\|} n$. However, we should not assume perfect equilibration of the ion temperature. This necessity to decouple electron and ion temperature is the reason why we chose to maintain separate ion and electron pressure evolution equations rather than assuming $p_{i} \propto p_{e}$.

We illustrate the existence of the ITG mode in our 6-field model by performing a Fourier analysis of our equation set in the local limit, i.e. assuming that all equilibrium terms, like

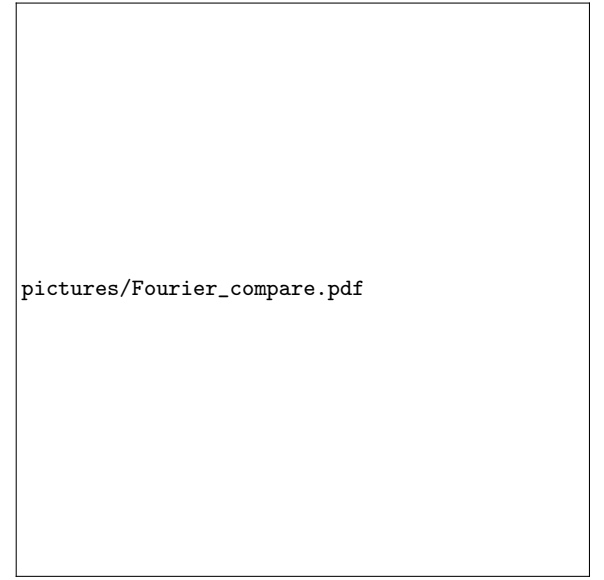

FIG. 2: Growth rates corresponding to the equilibrium gradients indicated by the red point in figure 1 , that is $R / L_{T}=25$ and $R / L_{n}=10$. The solid lines correspond to the six branches obtained from Fourier analysis. The symbols indicate the growth rates obtained using our initial value code Monja.

$v^{*}(x)$, are constant in space. In slab geometry, the resulting dispersion relation does not depend on the magnetic shear. We compute the growth rate of the most unstable branch at $k_{y} \rho_{i}=0.4$ for various values of the density and pressure gradients. We obtain a typical ITG instability diagram as shown in Figure 1. The parameters for this simulation correspond to the local plasma parameters at the $q=2$ resonant surface of the recent plasma discharge \#53259 of the WEST tokamak, that is a toroidal field of $3.6 \mathrm{~T}$, electron density of $1.8 \times 10^{19} \mathrm{~m}^{-3}$ and electron temperature of $T_{e}=1 \mathrm{keV}$. The ion temperature ratio is $\tau_{i}=T_{i} / T_{e}=0.32$. The local gradients are given by $R / L_{T}=25$ and $R / L_{n}=10$.

We choose the remaining free parameter, the perpendicular scale length $L_{\perp}$, to equal one tenth of the radial position of the $q=2$ resonant surface $r=0.3 \mathrm{~m}$. The resulting dimensionless simulation parameters are $\Omega \tau_{A}=0.43$, $\rho_{*}^{2}=0.0036, \kappa_{1}=0.011$ and $\kappa_{2}=0.09$. The equilibrium profiles are characterised by $v^{*}=-0.0043$ for the total pressure and $\partial_{x} N_{e q}=-0.13$ for the density. The equilibrium profiles for the parallel velocity $u_{\|}$and the electrostatic potential $\phi$ are set to zero. For the dissipative parameters we choose $D=\eta=\mu=\chi_{\perp e}=\chi_{\perp i}=10^{-6}$

To underline the ITG features we took the limit of a very high parallel electron heat flux $\left(\chi_{\| e}=10^{3}\right)$ while setting the parallel ion heat flux to zero. In the absence of magnetic shear the parameter $\psi_{e q}^{\prime}$ is a constant that reflects the strength of the parallel derivative, $\nabla_{\|} \widehat{=} \psi_{e q}^{\prime} \partial_{y}$. We choose $\psi_{e q}^{\prime}=0.003$ to obtain reasonable growth rates. Figure 1 shows the resulting growth rates expressed in terms of the ion thermal speed $v_{t h, i}=\sqrt{T_{i} / m_{i}}$ in dependence of the density and temperature 


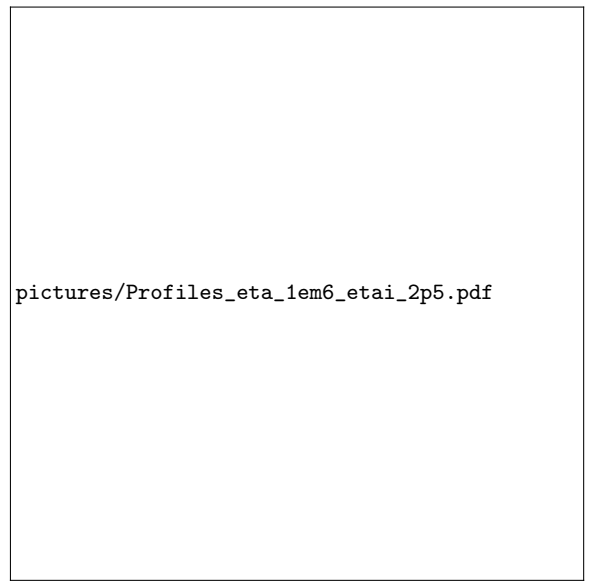

FIG. 3: The equilibrium profiles $\psi_{e q}^{\prime}(x)=\tanh (x / a)$ and $p_{e q}(x)=p_{0 N}\left(1-\frac{1}{4} \tanh \left(x / a_{P}\right)\right)$ for the case $R / L_{n}=10$, $R / L_{T}=25$ including a magnetic shear of $s=1.49$.

gradients. We obtain a roughly parabola-shaped unstable region that is typical for a fluid model of the ITG mode.

The gradients corresponding to the WEST discharge are marked by a red point. For this case, the growth rates are explicitly shown as a function of the poloidal wave number in figure 2 . We see that the most unstable mode is found at $k_{y} \rho_{i} \simeq 0.4$, coherent with results found for various fluid and gyrokinetic simulations of the ITG mode ${ }^{48}$. On the same graph we also show the growth rates obtained using our initial value code Monja for the same parameters. Both computational methods yield identical growth rates for all unstable modes, except for the largest-scale modes where the local approximation is no longer valid, and stable modes for which the initial-value approach is irrelevant.

\section{ITG mode with magnetic shear}

We now want to identify the ITG instability in the presence of magnetic shear using our code Monja. For all numerical simulations we use a resolution of $N_{x}=513$ times $N_{y}=256$ grid points for a simulation box of $L_{x}=\pi$ and $L_{y}=5 \pi$. In contrast to the Fourier analysis we cannot use arbitrarily large parallel heat fluxes in numerical simulations since the required temporal resolution increases drastically. We choose $\chi_{\| e}=100$ and $\chi_{\| i}=\chi_{\| e} \sqrt{m_{e} / m_{i}}$. We use the Harris ${ }^{49}$ current sheet model $\psi_{e q}^{\prime}(x)=\tanh (x / a)$ to describe the magnetic field in the vicinity of a resonant surface. For the $q=2$ resonant surface of the considered discharge the magnetic shear is $s=1.49$. This corresponds to a magnetic shear length of $a=148$, which is very large compared to the simulation box size. In this configuration the tearing mode

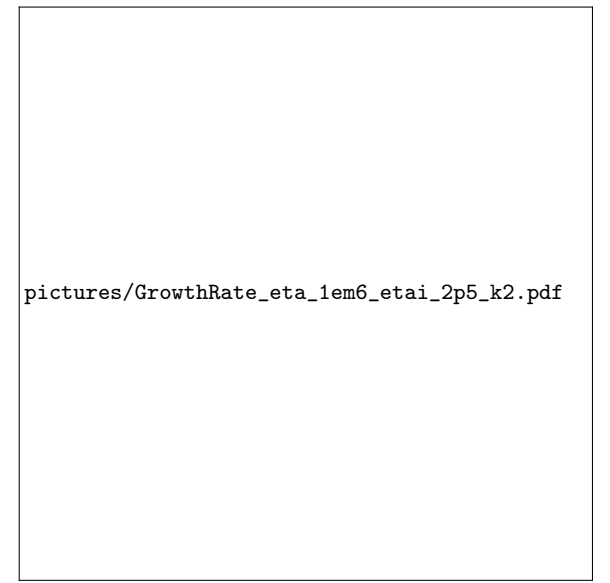

FIG. 4: When magnetic shear is included, we find much lower growth rates than in figure 2 . The most unstable mode corresponds now to $m=k_{y} L_{y}=16$.

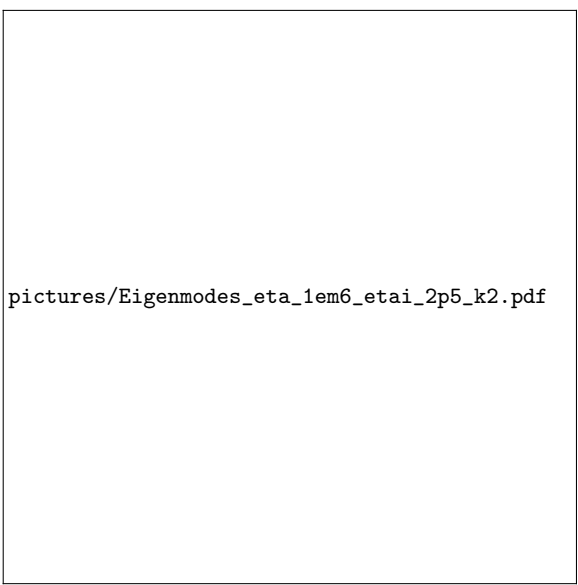

FIG. 5: Eigenmodes of the most unstable mode $m=16$ for the simulation shown in figure 4.

is linearly stable, since the tearing stability index parameter $\Delta^{\prime}=-1.4$ is negative. The equilibrium pressure profile is modelled by a hyperbolic tangent with limited amplitude: $p_{e q}(x)=p_{0 N}\left(1-\frac{1}{4} \tanh \left(x / a_{P}\right)\right)$. We set $a_{P}=-p_{0 N} /\left(4 v^{*}\right)$ so that the pressure gradient at the resonance is equal to $v^{*}$. The density equilibrium profile is also a hyperbolic tangent with the same width, so that the resulting equilibrium temperature profile is a smooth, monotonic function of $x$. The equilibrium magnetic and pressure profiles are shown in figure 3 .

The magnetic shear strongly stabilizes the ITG mode. As 


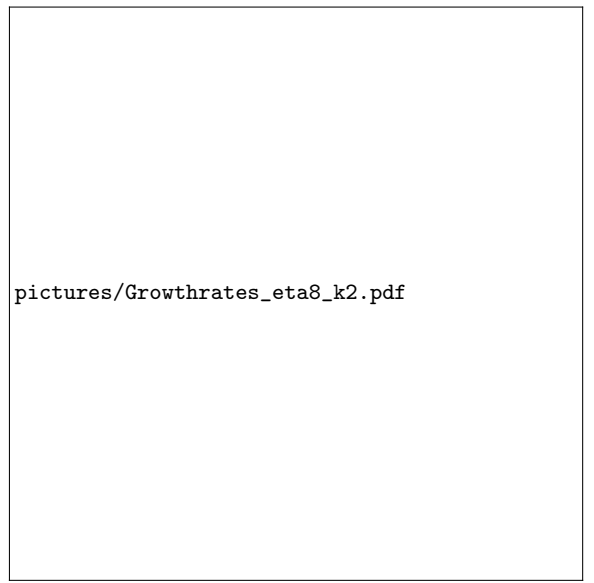

FIG. 6: Maximal growth rate of the most unstable mode as a function of the ITG stability parameter $\eta_{i}=L_{n} / L_{T}$ ( $\eta$ is the resistivity). This result was obtained using the initial value code and inclusing magnetic shear.

can be seen from figure 4 , the growth rates are about one order of magnitude lower than in the case without magnetic shear Also the most unstable mode is now located at $k_{y} \rho_{i} \approx 0.15$, which corresponds to the poloidal mode number $m=16$ : parallel damping is stronger on small-scale modes. Its mode structure is shown in figure 5 . The magnetic potential $\psi$ shows odd parity while the parity of the electrostatic potential $\phi$ is approximately even, apart from a slight shift to the right of the resonant surface that is due to the asymmetric equilibrium profiles. We recover thus the parity signature that is typical for an interchange-type instability.

To verify that this instability is indeed an ITG mode, we vary the ratio $\eta_{i}=L_{n} / L_{T}$ while keeping $R / L_{p}=R / L_{T}+$ $R / L_{n}=35$ constant. This corresponds to a scan along the red line in figure 1 . The case $R / L_{n}=10, R / L_{T}=25$ corresponds to $\eta_{i}=2.5$. For each simulation we determine the growth rate of the most unstable mode. Figure 6 shows that the instability appears as $\eta_{i}$ exceeds a critical value of about one. This is consistent with the instability criterion (33) which predicts the ITG mode to turn unstable if $\eta_{i}>1.5$ for the chosen parameters. However, at $\eta_{i}<1$ we observe another instability at smaller poloidal mode numbers. In the following we will characterise the different nature of those two instabilities at large and low $\eta_{i}$.

First, we decrease the resistivity down to $\eta=10^{-8}$. The results are also shown in figure 4 . The growth rates for $\eta_{i}>1$ depend very little on $\eta$, as it is expected for an ITG mode. In contrast, the mode at small $\eta_{i}$ is strongly stabilised.

Second, we consider the relative amplitudes and phase differences of the density and the electron and ion temperature fluctuations with respect to the electrostatic potential $\phi$, always for the most unstable mode. The amplitudes, shown in

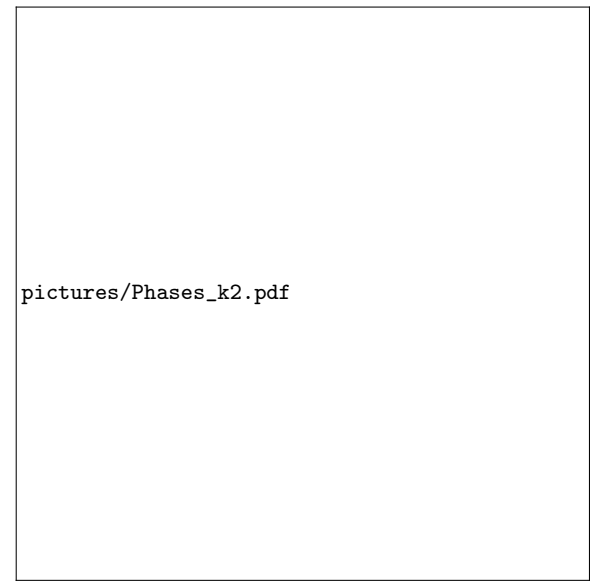

FIG. 7: Phase differences for the most unstable mode in dependence of the ITG stability parameter $\eta_{i}=L_{n} / L_{T}$.

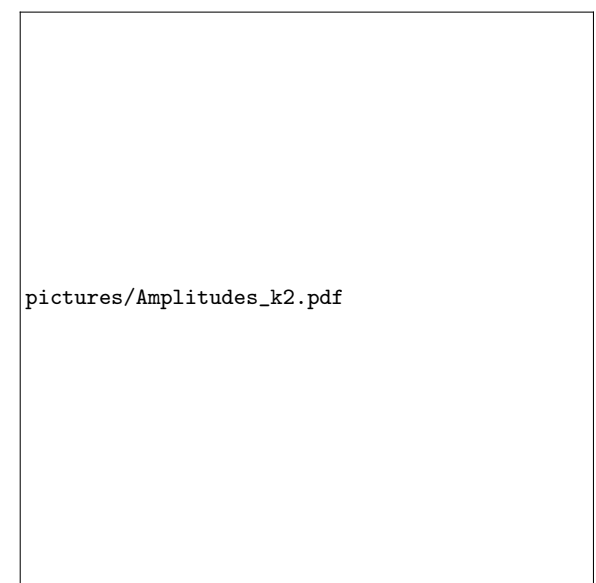

FIG. 8: Amplitude ratios for the most unstable mode in dependence of the ITG stability parameter $\eta_{i}=L_{n} / L_{T}$.

figure 8 , are divided by $p_{0 N}$ for the fields $T_{i}, T_{e}$ and $\phi$, but not for $n$ to account for our specific choice of the normalisation. The phase differences in figure 7 were evaluated within the interval defined by the radial positions of the maximal amplitude of the four fields $\phi, T_{i}, T_{e}$ and $n$. Variations of the phase differences within this interval are reflected by the error bars. We observe that the instability at small $\eta_{i}$ is dominated by density fluctuations, while for $\eta_{i}>1$ the fluctuations of the ion temperature are dominant. The phase shift between the density fluctuations and the fluctuations of $\phi$ is always small, indicating very little density transport. This is consistent with 


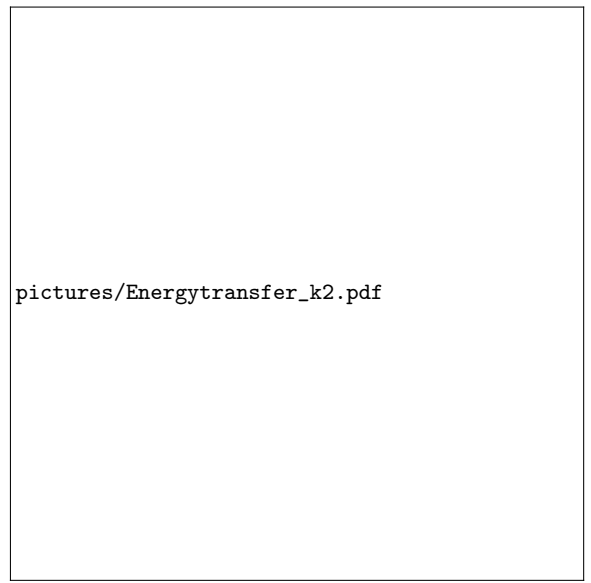

FIG. 9: Energy transfer rates between electrons, ions and the electromagnetic field. in dependence of the ITG stability parameter $\eta_{i}=L_{n} / L_{T}$. To convert from the Alfvén time to the thermal ion speed use $\tau_{A}=1.3 \cdot 10^{-4} v_{t h_{i}} / R$

an instability involving quasi adiabatic electrons. On the other hand, for the mode $\eta_{i}>1$ the phase shift between temperature and $\phi$ fluctuations is close to $\pi / 2$, maximizing the extraction of free energy from the temperature gradient. There is therefore a strong transport of the ion temperature and, interestingly, of the electron temperature as well.

Third, we study the contribution of the ion and electron species to the mode energy. In figure 9 we show the energy transfer rates $\delta E_{i \rightarrow f}, \delta E_{e \rightarrow f}$ and $\delta E_{i \rightarrow e}$ as defined in equations (28), (27) and (25). For the modes at low $\eta_{i}$ the electrons transfer most energy to the field, while the ions contribute very little. The energy transfer between the two species is very small, with the ions receiving energy from the electrons. Fo the modes at high $\eta_{i}$ the situation is opposite. It is the ions that transfer energy to the field and thus drive the mode unstable. The electrons give little energy to the field, but take some energy from the ions.

From these observations we conclude that the instability observed for $\eta_{i}>1$ is indeed an ITG mode, since it is driven by the ion dynamics, destabilised by $\eta_{i}$ and largely independent of resistivity. It is completely stabilised if the equilibrium ion temperature ratio $\tau_{i}$ is set to zero.

Conversely, the instability at small $\eta_{i}$ is not consistent with an ITG or curvature-destabilized $\eta_{i}$ mode ${ }^{50,51}$ : it is driven by electron dynamics, as shown by Fig.9, and is stabilized by increasing $\eta_{i}$. However, it is consistent with a propagating drift wave, slightly destabilized by resistivity ${ }^{52}$. Indeed, in adddition to being driven by the density gradient, its real frequency $\mathscr{R} e(\omega)=9.01 v_{t h, i} / R$ matches the expected frequency for a drift wave $k_{\theta} \rho_{i} R / L_{n}=8.49 v_{t h, i} / R$, with $k_{\theta} \rho_{i}=0.24$ for the most unstable mode (Figure 6, leftmost blue circle). The growth rate itself is about 20 times lower than the real fre-

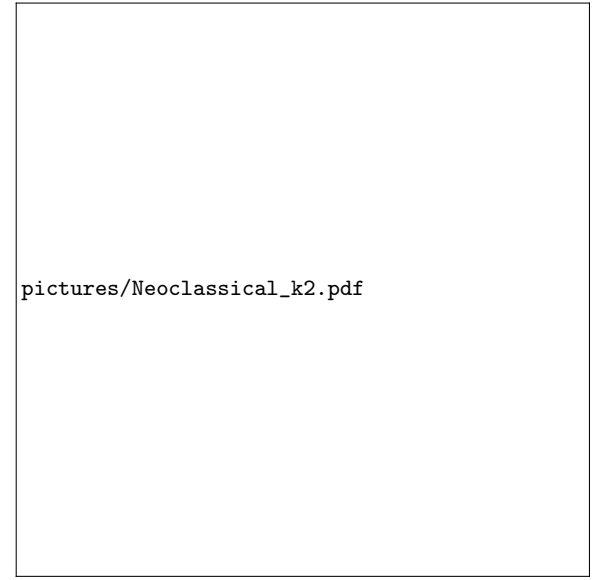

FIG. 10: Impact of neoclassical terms on the maximal growth rate of the ITG mode at $\eta_{i}=10$. The most unstable mode number is $m=26$ for the first data point, $m=25$ for the others.

quency, as expected for a mostly propagating wave, and the phase shift between $\phi$ and $n$ fluctuations is small.

After having identified the ITG mode in our model, we complete its characterisation by determining how it is impacted by neoclassical physics. For this study we use the case $\eta_{i}=10$. When adding the neoclassical terms we need to consider the additional constraint on the equilibrium terms $u_{\theta, e q}=-k_{i} \partial_{x} T_{i, e q}$ that is induced by neoclassical friction. We first choose to maintain $u_{\| e q}=0$ and to adapt the electrostatic potential so that $\phi_{e q}(x)=\phi_{n c, e q}(x)=-p_{i, e q}(x)-k_{i} T_{i, e q}(x)$. The impact of $u_{\| e q}$ is studied below. For the parameters related to the neoclassical terms we use $\varepsilon_{g}=0.13, q=2, k_{i}=-0.3$, $k_{e}=-0.74$ and $\mu_{i}^{n c}=10^{-6}$.

As expected, the neoclassical terms do not drastically change the linear growth rate of the ITG mode. Their main impact is the modification of the equilibrium flow. In figure 10 we show the maximal growth rate first for the case without neoclassical terms nor a neoclassical equilibrium flow. Then we add just the neoclassical equilibrium flow $\phi_{e q, n c}$ without neoclassical terms, which considerably reduces the growth rate. In fact $\phi_{e q, n c}$ represents a sheared $\mathrm{E} \times \mathrm{B}$ flow that is known to have a stabilising effect. Next we add just the boostrap current terms $J_{b s}$, which is barely destabilising. Finally we include the remaining neoclassical terms which are friction terms proportional to $\mu_{i}^{n c}$. Interestingly these terms are also destabilising. The remaining properties that we have used to identify our mode as an ITG mode, like phase shifts and amplitude ratios, are very little influenced by the neoclassical terms and the modified equilibrium. We observe deviations of a few per cent. Only the energy transfer rate from the electrons to the field is four times higher when neoclassical terms are included, but the energy contribution of the electrons to 


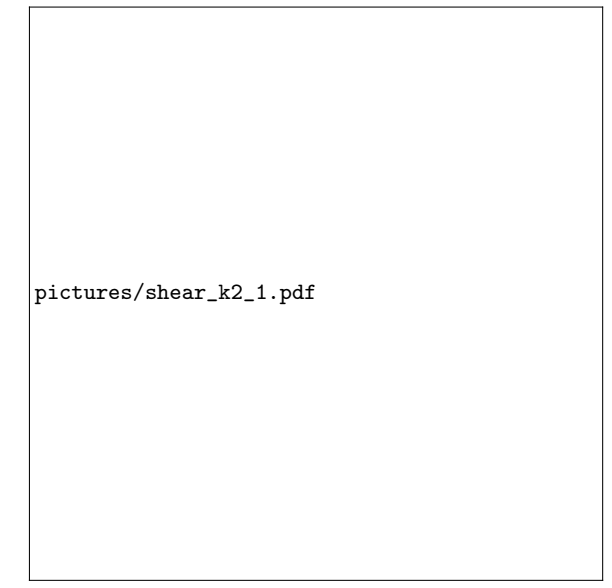

FIG. 11: The ITG mode with neoclassical terms shown in figure 10 is destabilised by imposing a flow shear on the equilibrium field $u_{\| e q}$. If the parallel dynamics is suppressed ( 5 fields, $\tilde{u}_{\|}=0$ ) we find stabilisation of the ITG mode by the flow shear. All results obtained with $\mu_{i}^{n c}=10^{-6}$ and $u_{\theta, e q}=-k_{i} \partial_{x} T_{i}$.

the mode remains still an order of magnitude lower than the ion contribution.

We state that the most important impact of the neoclassical terms on the ITG properties is the reduction of the linear growth rate by the sheared flow induced by the constraint $u_{\theta, e q}=-k_{i} \partial_{x} T_{i, e q}$ on the equilibrium. So far we have fulfilled this condition by choosing $\partial_{x} u_{\| e q}=0$ and adapting $\phi_{e q}=-p_{i}-k_{i} T_{i}$. Of course we are free to choose a non-zero flow equilibrium $u_{\|, e q}$. We are interested in the impact of a radial flow shear $\partial_{x} u_{\|, e q}$ on the linear growth rate of the most unstable mode. Because of the constraint $u_{\theta, e q}=-k_{i} \partial_{x} T_{i, e q}$, this results in a sheared $\mathrm{E} \times \mathrm{B}$ flow $\partial_{x x} \phi_{e q}$. The linear growth rate of a toroidal ITG mode is expected to decrease with increasing shear flow ${ }^{52-54}$, while the slab ITG mode may be further destabilised by moderate shear flow ${ }^{55}$. In figure 11 we show the impact of the equilibrium flow shear on our 6field ITG model for the case $\eta_{i}=10$ with neoclassical terms. Since our ITG model contains both toroidal (curvature) and slab $\left(u_{\|}\right)$ITG characteristics, we show also the results for a pure toroidal ITG that we obtain by setting $\tilde{u}_{\|}=0$, thus suppressing the parallel dynamics.

We observe that the purely toroidal 5-field ITG mode can be completely stabilised by the flow shear. In a toroidal geometry, this can be attributed to the reduction in the radial width of the mode structure ${ }^{53}$. However, in our case with uniform curvature, we do not observe this radial width reduction. But we do observe an increasing radial displacement of the eigenfunctions away from the resonant surface as the shear flow increases ${ }^{56}$. This has a stabilising effect since dissipative terms in our model increase with the distance to the resonan

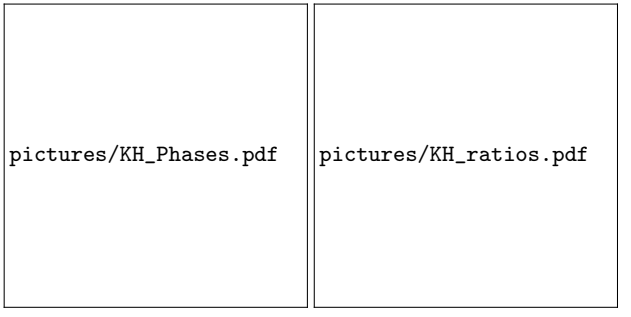

(a)

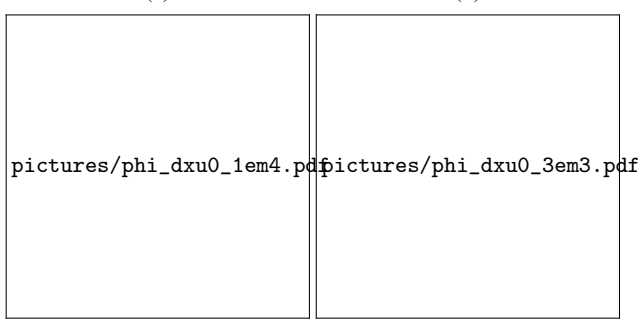

(c)

(d)

FIG. 12: Destabilization by the parallel velocity gradient in the 6-field model. Phase shift (a), fluctuation amplitude ratio (b), and mode structure below (c) and above (d) threshold.

surface.

The full 6-field ITG mode is strongly destabilised by a weak flow shear. For the slab ITG mode, as explained in ${ }^{55}$, the parallel compression terms $\nabla_{\|} \boldsymbol{u}_{\|}$that couples the parallel dynamics to the remaining fields represents an instability drive that is strongest at large distances from the resonant surfaces. Indeed, as the equilibrium flow shear increases above the threshold $\partial_{x} u_{\|, e q}>c_{s} / L_{n}\left(\simeq 0.7 \cdot 10^{-3} \tau_{A}^{-1}\right.$ in our simulations $)$, we observe that the instability source progressively shifts from the temperature gradient to the parallel velocity gradient. This is shown on figure 12. First, at low flow shear the phase shift with respect to potential fluctuations is optimal $(\pi / 2)$ for $\tilde{T}_{i}$ fluctuations, while at high flow shear it is optimal for $\tilde{u}_{\|}$fluctuations (the opposite sign comes from the fact that $\partial_{x} T_{i, e q}<0$ while $\partial_{x} u_{\|, e q}>0$ ). Second, the ratio of parallel velocity fluctuations to ion temperature fluctuations is constant below the threshold, and increases above the threshold. Third, above the threshold the maxima of the eigenmode amplitudes are progressively shifted radially, away from the resonance where $\nabla_{\|, e q}=0$; this increases the destabilising impact of $\nabla_{\|} u_{\|}$.

\section{CONCLUSION}

We have derived a six-field reduced MHD model that describes both ITG turbulence and the tearing mode, including neoclassical effects. The model allows the construction of an energy-like quantity with a linear pressure contribution that is conserved in the limit of no dissipations, vanishing Lar- 
mor radius and neglect of the neoclassical terms. We have demonstrated the existence of the ITG mode in our model first by analytically reducing our model to a known 2-field ITG model, then by eigenvalue and initial value numerical simulations with and without magnetic shear. Neglecting the neoclassical terms, we showed that, as expected, the ITG mode is destabilised when the ratio $\eta_{i}=L_{n} / L_{T}$ exceeds a critical value of about one and that its growth rate is independent of the plasma resistivity. Finally we verified that adding the neoclassical terms does not substantially alter the linear stability properties of the ITG mode. We recover the familiar shear flow stabilisation of a toroidal ITG mode while respecting the neoclassical constraints on the equilibrium poloidal velocity.

We have thus obtained a model that may be used to study the nonlinear interaction between ITG turbulence and a neoclassical tearing mode. In the future we intend to furthe expand this model to describe the turbulent and neoclassical transport of one trace impurity species. This will allow us studying the impact of a neoclassical tearing mode on impurity transport.

\section{ACKNOWLEDGMENTS}

The authors wish to thank Andrei Smolyakov for fruitful discussions. This work was granted access to the HPC resources of Aix-Marseille Université financed by the project Equip@Meso (ANR-10-EQPX-29-01) of the program 'Investissements d'Avenir' supervised by the Agence Nationale de la Recherche. It has been also carried out within the framework of the EUROfusion Consortium and French Research Federation for Fusion Studies and has received funding from the Euratom research and training programme 2014-2018 and 2019-2020 under grant agreement No. 633053. The views and opinions expressed herein do not necessarily reflect those of the European Commission. Some simulations were performed with the support of EUROfusion and MARCONI-Fusion.

\section{APPENDIX 1: VORTICITY EQUATION}

Here we present the derivation of the vorticity equation (8) in detail. The remaining evolution equations are obtained in a similar manner, but their derivation is less complex. We start by writing the sum of the momentum equations (2) for electrons and ions:

$$
\begin{aligned}
n m_{i}\left(\partial_{t}+(\mathbf{u} \cdot \nabla)\right) \mathbf{u}= & \mathbf{J} \times \mathbf{B}-\nabla\left(p_{i}+p_{e}\right) \\
& -\nabla \cdot\left(\overline{\bar{\Pi}}_{i}+\overline{\bar{\Pi}}_{e}\right)+\mathbf{R}_{i}+\mathbf{R}_{e}
\end{aligned}
$$

Since the momentum exchange of electrons with ions is equal to minus the momentum exchange of ions with electrons, we have $\mathbf{R}_{i}+\mathbf{R}_{e}=0$ according to ${ }^{32}$. We express the plasma velocity $\mathbf{u}$ in terms of the parallel velocity and the drift velocities using equation (4). For ease of notation we replace the sum of all perpencicular drift velocities by $\mathbf{u}_{\perp}$. We use the diamagnetic cancellation according to ${ }^{33}$ equation (34) to remove the advection by the diamagnetic drift velocity:

$$
\begin{aligned}
& n m_{i}\left(\partial_{t}+\left(\left(u_{\|} \mathbf{b}+\mathbf{u}_{E \times B}\right) \cdot \nabla\right)\right)\left(u_{\|} \mathbf{b}+\mathbf{u}_{\perp}\right)=\mathbf{J} \times \mathbf{B} \\
& -\nabla\left(p_{i}+p_{e}\right)-\nabla \cdot\left(\overline{\bar{\Pi}}_{i}-\overline{\bar{\Pi}}_{i}^{g v}+\overline{\bar{\Pi}}_{e}-\overline{\bar{\Pi}}_{e}^{g v}\right)-\nabla \chi^{\prime}
\end{aligned}
$$

The cancellation involves a part of the non-diagonal gyroviscous stress tensor $\overline{\bar{\Pi}}^{g v}$ as well as a scalar function $\chi^{\prime}$. The remaining non-diagonal part or the stress tensor results approximatively in the dissipative term $\mu \Delta_{\perp}^{2} \phi$ that we will reinsert at the last step. For the diagonal part of the stress tensor we will use a neoclassical closure relation. Since the contribution for ions is $\sqrt{m_{i} / m_{e}}$ times larger than the one for electrons, we neglect $\bar{\Pi}_{e}$. We now project this equation to the perpendicular direction by taking the rotational and multiplying by $\mathbf{B}$ :

$$
\begin{aligned}
\mathbf{B} \cdot\left(\nabla \times n m_{i} \mathrm{~d}_{t}^{0} \mathbf{u}_{\perp}\right)= & \mathbf{B} \cdot(\nabla \times(\mathbf{J} \times \mathbf{B})) \\
& -\mathbf{B} \cdot\left(\nabla \times \nabla \cdot \overline{\bar{\Pi}}_{i}\right)
\end{aligned}
$$

Here we have introduced the definition $\mathrm{d}_{t}^{0}=\partial_{t}+\mathbf{u}_{E \times B}$. $\nabla+u_{\|} \nabla_{\|}$. The remaining $u_{\|}$term has vanished due to the projection perpendicular to $\mathbf{B}$, and the gradient terms $\nabla p$ and $\nabla \chi^{\prime}$ have disappeared because the rotation of a gradient is always zero. To evaluate the momentum term on the left hand side, we first observe that it is $\mathscr{O}\left(\varepsilon_{w}\right)=\mathscr{O}\left(\varepsilon_{g}^{2}\right)$ smaller than the dominant terms on the right hand side. Since we will neglect all terms of order $\varepsilon^{3}$, we need to retain only the leading order terms. We therefore replace the magnetic field by $\mathbf{B}_{e q}$ and the density by its constant average value:

$$
m_{i} \mathbf{B} \cdot\left(\nabla \times n \mathrm{~d}_{t}^{0} \mathbf{u}_{\perp}\right)=m_{i} n_{0} \mathrm{~d}_{t}^{0}\left(\mathbf{B}_{e q} \cdot\left(\nabla \times \mathbf{u}_{\perp}\right)\right)+\mathscr{O}\left(\varepsilon^{3}\right)
$$

The rotational can be interchanged with the total derivative $\mathrm{d}_{t}^{0}$ to leading order in $\varepsilon_{g}$. Following the same argumentation we retain only the two dominant drift terms, $\mathbf{u}_{E}$ and $\mathbf{u}_{p}^{*}$.

$$
\begin{aligned}
m_{i} \mathbf{B} & \cdot\left(\nabla \times n \mathrm{~d}_{t}^{0} \mathbf{u}_{\perp}\right) \\
& =m_{i} n_{0} \mathrm{~d}_{t}\left(\mathbf{B}_{e q} \cdot\left(\nabla \times\left(\mathbf{u}_{E}+\mathbf{u}_{p}^{*}\right)\right)\right)+\mathscr{O}\left(\varepsilon^{3}\right) \\
& =m_{i} n_{0} \mathrm{~d}_{t}\left(\Delta_{\perp} \phi+\nabla_{\perp} \frac{\nabla p_{i}}{e n}\right)+\mathscr{O}\left(\varepsilon^{3}\right) \\
& =m_{i} n_{0} \mathrm{~d}_{t} \Delta_{\perp}\left(\phi+\frac{p_{i}}{e n_{0}}\right)+\mathscr{O}\left(\varepsilon^{3}\right)
\end{aligned}
$$

The calculation of the first term on the right hand side of equation (35) yields:

$$
\begin{aligned}
\mathbf{B} \cdot(\nabla \times(\mathbf{J} \times \mathbf{B})) & =B^{2} \nabla_{\|} J_{\|}+B^{2} \boldsymbol{J}_{\|} \mathbf{b} \cdot \frac{\nabla B}{B}-2 B^{2} \mathbf{J} \cdot \frac{\nabla B}{B} \\
& \approx B^{2} \nabla_{\|} \boldsymbol{J}_{\|}-2 B^{2} \frac{\nabla B}{B} \cdot \mathbf{J}_{\perp}
\end{aligned}
$$

We use the identity $\mathbf{J}_{\perp}=(\mathbf{B} \times(\mathbf{J} \times \mathbf{B})) / B^{2}$ to express $\mathbf{J}_{\perp}$ with the help of equation (34) where we neglect the inertia term:

$$
\begin{gathered}
2 B^{2} \frac{\nabla B}{B} \cdot \mathbf{J}_{\perp}=2 \frac{\nabla B}{B} \cdot\left(\mathbf{B} \times\left(\nabla\left(p_{i}+p_{e}\right)+\nabla \cdot \overline{\bar{\Pi}}_{i}\right)\right) \\
=2\left\{p_{i}+p_{e}, B\right\}+2 \frac{\nabla B}{B} \cdot\left(\mathbf{B} \times \nabla \cdot \overline{\bar{\Pi}}_{i}\right)
\end{gathered}
$$


Inserting the results (36) to (38) into (35) gives:

$$
\begin{gathered}
m_{i} n_{0} \mathrm{~d}_{t} \Delta_{\perp}\left(\phi+\frac{p_{i}}{e n_{0}}\right)=B^{2} \nabla_{\|} \boldsymbol{J}_{\|}-2\left\{p_{i}+p_{e}, B\right\} \\
-2 \frac{\nabla B}{B}\left(\mathbf{B} \times \nabla \cdot \overline{\bar{\Pi}}_{i}\right)-\mathbf{B} \cdot\left(\nabla \times \nabla \cdot \overline{\bar{\Pi}}_{i}\right)
\end{gathered}
$$

We introduce the generalised vorticity $\omega=\Delta_{\perp}\left(\phi+p_{i} /\left(e n_{0}\right)\right)$ to write the equation in a more compact form. The advection part of the total time derivative can conveniently be expressed in terms of the Poisson bracket:

$$
\begin{aligned}
\mathrm{d}_{t}^{0} \omega & =\partial_{t} \omega+\frac{\mathbf{B} \times \nabla \phi}{B^{2}} \cdot \nabla \omega+u_{\|} \nabla_{\|} \omega \\
& =\partial_{t} \omega+\frac{1}{B}\{\phi, \omega\}+u_{\|} \nabla_{\|} \omega
\end{aligned}
$$

We neglect the parallel advection of the vorticity since this is a higher-order (cubic) term. Finally we reinsert the dissipative term $+\mu /\left(n_{0} m_{i}\right) \Delta_{\perp} \tilde{\boldsymbol{\omega}}$ from the non diagonal part of the stress tensor. We obtain thus the vorticity equation (8).

\section{APPENDIX 2: GYROVISCOUS CANCELLATION}

In the pressure equation we use the gyroviscous cancellation as given in $^{57}$, which is based on the expression of the heat flux $\mathbf{q}$ as given by Braginski ${ }^{32}$.

$$
\mathbf{q}_{s}=\mathbf{q}_{\| s}+\mathbf{q}_{\perp s}+\mathbf{q}_{\wedge s} \quad \text { with } \quad \mathbf{q}_{\wedge s}=\frac{5}{2} \frac{n T_{s}}{e_{s} B} \mathbf{b} \times \nabla T_{s}
$$

From this definition we obtain straightforwardly:

$$
\begin{aligned}
& \frac{5}{2} \nabla \cdot\left(\frac{\mathbf{B}}{e_{s} B^{2}} \times \nabla\left(p_{s} T_{s}\right)\right) \\
& =\frac{5}{2} \nabla \cdot\left(\frac{T_{s}}{e_{s} B^{2}} \mathbf{B} \times \nabla p_{s}\right)+\frac{5}{2} \nabla \cdot\left(\frac{p_{s}}{e_{s} B^{2}} \mathbf{B} \times \nabla T_{s}\right) \\
& =\frac{5}{2} \nabla \cdot\left(p_{s} \mathbf{u}_{p_{s}}^{*}\right)+\nabla \cdot \mathbf{q}_{\wedge s} \\
& =\frac{5}{2} \mathbf{u}_{p_{s}}^{*} \cdot \nabla p_{s}+\frac{5}{2} p_{s} \nabla \cdot \mathbf{u}_{p_{s}}^{*}+\nabla \cdot \mathbf{q}_{\wedge s}
\end{aligned}
$$

We have used the expression of the diamagnetic drift velocity $\mathbf{u}_{p_{s}}^{*}=\frac{\mathbf{B} \times \nabla p_{s}}{e_{s} n B^{2}}$. From this expression we also see that the term $\mathbf{u}_{p_{s}}^{*} \cdot \nabla p_{s}$ is actually zero, therefore we can conveniently change its pre-factor to obtain the desired identity:

$$
\frac{3}{2} \mathbf{u}_{p_{s}}^{*} \cdot \nabla p_{s}+\frac{5}{2} p_{s} \nabla \cdot \mathbf{u}_{p_{s}}^{*}+\nabla \cdot \mathbf{q}_{\wedge s}=\frac{5}{2} \nabla \cdot\left(\frac{\mathbf{B}}{e_{s} B^{2}} \times \nabla\left(p_{s} T_{s}\right)\right)
$$

\section{APPENDIX 3: NEOCLASSICAL TERMS}

Here we give details on the calculation of our neoclassical terms that result from the stress tensor $\overline{\bar{\Pi}}_{s}$. The terms we need to calculate appear in the following four equations:

$$
\begin{aligned}
& \partial_{t} \psi=\ldots- \frac{\mathbf{b} \cdot\left(\nabla \cdot \overline{\bar{\Pi}}_{e}\right)}{e n_{0}} \\
& \partial_{t} \omega=\ldots-\frac{2}{m_{i} n_{0}} \frac{\nabla B}{B} \cdot\left(\mathbf{B} \times \nabla \cdot \overline{\bar{\Pi}}_{i}\right)-\frac{\mathbf{B}}{m_{i} n_{0}} \cdot\left(\nabla \times \nabla \cdot \overline{\bar{\Pi}}_{i}\right) \\
& \partial_{t} p_{i}=\ldots+\frac{5}{3} \frac{p_{i}}{e n_{0} B^{2}}\left(\frac{\nabla B}{B} \cdot\left(2 \mathbf{B} \times\left(\nabla \cdot \overline{\bar{\Pi}}_{i}\right)\right)\right. \\
&\left.\quad+\mathbf{B} \cdot\left(\nabla \times\left(\nabla \cdot \overline{\bar{\Pi}}_{i}\right)\right)\right) \\
& \partial_{t} u_{\|}=\ldots-\frac{\mathbf{b} \cdot\left(\nabla \cdot \overline{\bar{\Pi}}_{i}\right)}{m_{i} n_{0}}
\end{aligned}
$$

\section{A. Closure on viscous force}

Here we calculate the neoclassical terms (39) using the closure (15) for the viscous force. The first step is to calculate the poloidal component of the plasma flow plus heat flux:

$$
\mathbf{e}_{\theta} \cdot \mathbf{v}_{s}=\mathbf{e}_{\theta} \cdot\left(\mathbf{u}_{s}+\frac{2}{5} k_{s} \frac{\mathbf{q}_{s}}{p_{s}}\right)
$$

This is done by using the decomposition (4) of the velocity and a Braginskii-type ${ }^{32}$ heat flux:

$$
\mathbf{q}_{s}=\mathbf{q}_{\| s}+\frac{5}{2} \frac{p_{s}}{e_{s}} \frac{\mathbf{B} \times \nabla T_{s}}{B^{2}}
$$

It is convenient to express the parallel heat flux $q_{\| s}$ in terms of a normalised heat defined by:

$$
u_{2 \| i}=\frac{2}{5} \frac{q_{\| i} B}{p_{i}}
$$

For the equilibrium magnetic field we use the form

$$
\mathbf{B}_{e q}=\frac{B_{0} R_{0}}{R}\left(\mathbf{e}_{\varphi}+\frac{r}{q R} \mathbf{e}_{\theta}\right)
$$

where the $\theta$-dependence is given by $R=R_{0}+r \cos (\theta)$. We neglect terms of order $\varepsilon_{g}^{2}$ to obtain:

$$
\mathbf{e}_{\theta} \cdot \mathbf{v}_{s}=\frac{r}{q R}\left(u_{\| s}+u_{2 \| s} \frac{k_{i}}{B}\right)+\frac{R}{B_{0} R_{0}} \partial_{r} \Phi_{s}
$$

For ease of notation, we use the definition $\Phi_{s}=\phi+$ $p_{s} /\left(e_{s} n_{0}\right)+k_{s} T_{s} / e_{s}$. This formulation is now used to calculate the neoclassical terms in (39). We average these terms over the poloidal coordinate $\theta$, since in the current version of our numerical code we use slab geometry that does not take into account the poloidal variation of the equilibrium magnetic field.

When taking the poloidal average $\langle\cdot\rangle$ we assume that radial variations $\partial_{r}$ of all our fields, e.g. $u_{\|}$or $\phi$, are much larger than $1 / r$. We also assume that poloidal variations of all fields are of higher order in $\varepsilon_{g}$ and can be neglected. We consider only the poloidal variations of the equilibrium magnetic field 
(41). We also use the poloidal dependence of the parallel heat flux which is given by neoclassical theory, see for example equation (9.12) in $^{58}$

$$
u_{2 \| s}=\left\langle u_{2 \| s}\right\rangle \frac{B^{2}}{\left\langle B^{2}\right\rangle}+\frac{q \partial_{r} T_{i}}{\varepsilon_{g} e_{i}}\left(\frac{B^{2}}{\left\langle B^{2}\right\rangle}-1\right)
$$

To leading order in $\varepsilon_{g}$, we obtain the following expressions:

$$
\begin{aligned}
\left\langle\mathbf{b} \cdot\left(\nabla \cdot \overline{\bar{\Pi}}_{e}\right)\right\rangle & =-\frac{\mu_{e}^{n c}}{v_{e}} e n_{0} \eta j_{\|}+m_{e} n_{0} \mu_{e}^{n c}\left(u_{\|}+\left\langle u_{2 \| e}\right\rangle \frac{k_{e}}{B_{0}}+\frac{q}{\varepsilon_{g}} \frac{1}{B_{0}}\left(\partial_{r} \Phi_{e}\right)\right) \\
\left\langle\mathbf{b} \cdot\left(\nabla \cdot \overline{\bar{\Pi}}_{i}\right)\right\rangle & =m_{i} n_{0} \mu_{i}^{n c}\left(u_{\|}+\left\langle u_{2 \| i}\right\rangle \frac{k_{i}}{B_{0}}+\frac{q}{\varepsilon_{g}} \frac{1}{B_{0}} \partial_{r} \Phi_{i}\right) \\
\left\langle\mathbf{B} \cdot\left(\nabla \times \nabla \cdot \overline{\bar{\Pi}}_{i}\right)\right\rangle & =m_{i} n_{0} \mu_{i}^{n c} B_{0} \frac{q}{\varepsilon_{g}}\left(\partial_{r} u_{\|}+\frac{k_{i}}{B_{0}} \partial_{r}\left\langle u_{2 \| i}\right\rangle+\frac{q}{\varepsilon_{g} B_{0}} \partial_{r}^{2} \Phi_{i}\right) \\
\left\langle\frac{\nabla B}{B} \cdot\left(\mathbf{B} \times \nabla \cdot \overline{\bar{\Pi}}_{i}\right)\right\rangle & =-m_{i} n_{0} \mu_{i}^{n c} \frac{B_{0}}{R_{0}} q\left[\left(\frac{1}{2 q}-\frac{1}{2}\right) u_{\|}+\frac{1}{2 q}\left\langle u_{2 \| i}\right\rangle+\frac{k_{i}}{B_{0}} \frac{q}{\varepsilon_{g}} \frac{\partial_{r} T_{i}}{e_{i}}+\left(\frac{1}{2 q}-\frac{3}{2}\right) \frac{q}{\varepsilon_{g}} \frac{\partial_{r} \Phi_{i}}{B_{0}}\right]
\end{aligned}
$$

By comparing expression (46) to (45), we see that the former is a factor of $\varepsilon_{g}$ smaller than the latter. Therefore we neglect the terms proportional to $\nabla B \cdot\left(\mathbf{B} \times \nabla \cdot \overline{\bar{\Pi}}_{i}\right)$ in the expression of the neoclassical terms for the ion pressure and vorticity equations. Note that only the average part of the parallel heat flux $\left\langle u_{2} \| s\right\rangle$ contributes, the anisotropic contibution in (42) vanishes after poloidal averaging. Since we assume $\left\langle u_{2} \|_{s}\right\rangle=0$, only the perpendicular heat flux component is finally retained. Inserting the results (43) to (45) into the equation set (39) yields, after normalisation, the neoclassical terms that we included in our set of final equations (18) to (23)

\section{B. Closure on pressure anisotropy}

We now present an alternative closure that is based on the Chew-Goldberg-Low ${ }^{40}$ form of the dominant, diagonal part of the gyroviscous stress tensor $\overline{\bar{\Pi}}_{s}$ :

$$
\overline{\bar{\Pi}}_{s}=\frac{3}{2} \pi_{\| s}\left(\mathbf{b b}-\frac{1}{3} \mathbb{I}\right)
$$

This approach reduces the problem to a scalar function, the pressure anisotropy $\pi_{\| s}=\frac{2}{3}\left(p_{\| s}-p_{\perp s}\right)$. The following useful identities, given also in ${ }^{59}$, follow directly from relation (47):

$$
\begin{aligned}
\mathbf{b} \cdot(\nabla \cdot \overline{\bar{\Pi}}) & =\mathbf{b} \cdot \nabla \pi_{\|}+\frac{3}{2} \pi_{\|} \nabla \cdot \mathbf{b} \\
\mathbf{B} \times(\nabla \cdot \overline{\bar{\Pi}}) & =\frac{3}{2} \pi_{\|} \mathbf{B} \times(\mathbf{b} \cdot \nabla) \mathbf{b}-\frac{1}{2} \mathbf{B} \times \nabla \pi_{\|} \\
\mathbf{B} \cdot \nabla \times(\nabla \cdot \overline{\bar{\Pi}}) & =\frac{3}{2} \nabla \pi_{\|} \cdot(((\mathbf{b} \cdot \nabla) \mathbf{b}) \times \mathbf{B})
\end{aligned}
$$

With these results the neoclassical terms (39) take the following form:

$$
\begin{aligned}
\partial_{t} \psi & =\ldots-\frac{\mathbf{b} \cdot \nabla \pi_{\| e}}{e n_{0}}-\frac{3}{2} \frac{\pi_{\| e} \nabla \cdot \mathbf{b}}{e n_{0}} \\
\partial_{t} \omega & \approx \ldots-\frac{1}{m_{i} n_{0}} \frac{1}{2}\left\{\pi_{\| i}, B_{e q}\right\} \\
\partial_{t} p_{i} & \approx \ldots+\frac{p_{i}}{e n_{0} B^{2}} \frac{5}{6}\left\{\pi_{\| i}, B_{e q}\right\} \\
\partial_{t} u_{\|} & =\ldots-\frac{\mathbf{b} \cdot \nabla \pi_{\| i}}{m_{i} n_{0}}-\frac{3}{2} \frac{\pi_{\| i} \nabla \cdot \mathbf{b}}{m_{i} n_{0}}
\end{aligned}
$$

In the vorticity and pressure equations we approximated the curvature term $(\mathbf{b} \cdot \nabla) \mathbf{b}$ with the alternative curvature $\nabla B / B$, which is equivalent up to second order in $\varepsilon_{g}$. With this approximation, the two contributions $\mathbf{B} \times\left(\nabla \cdot \overline{\bar{\Pi}}_{i}\right)$ and $\nabla \times\left(\nabla \cdot \overline{\bar{\Pi}}_{i}\right)$ combine into just one neoclassical term. We notice already one important difference to the previous closure on the viscous force: Here, $\mathbf{B} \times\left(\nabla \cdot \overline{\bar{\Pi}}_{i}\right)$ cannot be neglected against $\nabla \times\left(\nabla \cdot \overline{\bar{\Pi}}_{i}\right)$, because both terms contribute equally.

We now need to introduce a closure relation on $\pi_{s}$. We use the standard formulation ${ }^{34}$, using the same $\mathbf{v}_{s}$ as above:

$$
\frac{3}{2} \pi_{|| s}=-\frac{n m_{s} \mu_{s} B^{2}}{\left\langle(\mathbf{b} \cdot \nabla B)^{2}\right\rangle} \mathbf{v}_{s} \cdot \frac{\nabla B}{B}
$$

After inserting this closure into (51), we obtain neoclassical terms that contain explicitly the poloidal variation of the equilibrium magnetic field. We calculate the poloidal average $\langle\cdot\rangle$ of all neoclassical terms, using the same approximations and the assumption $\left\langle u_{2 \mid s}\right\rangle=0$ as in the previous section. The terms $\mathbf{b} \cdot \nabla \pi_{\| s}$ vanish naturally after averaging. After normal- 
isation we obtain straightforwardly:

$$
\begin{aligned}
& \partial_{t} \psi=\ldots+\frac{\mu_{e}^{n c}}{v_{e}} \eta J_{\|}-\left(1+\frac{\mu_{e}^{n c}}{v_{e}}\right) J_{b s} \\
& \partial_{t} \omega=\ldots+\frac{1}{3} \mu_{i}^{n c}\left(\frac{q}{\varepsilon_{g}}\right)^{2}\left(\partial_{x} u_{\theta}+k_{i} \partial_{x} T_{i}\right) \\
& \partial_{t} p_{i}=\ldots-\frac{1}{3} \mu_{i}^{n c} \rho_{*}^{2} \frac{\tau_{i}}{1+\tau_{i}}\left(\frac{q}{\varepsilon_{g}}\right)^{2}\left(\partial_{x} u_{\theta}+k_{i} \partial_{x} T_{i}\right) \\
& \partial_{t} u_{\|}=\ldots-\mu_{i}^{n c} \frac{q}{\varepsilon_{g}}\left(u_{\theta}+k_{i} \partial_{x} T_{i}\right)
\end{aligned}
$$

For Ohm's law and the parallel velocity equation the results obtained with the two approaches are identical, but the neoclassical terms in the vorticity and pressure equations differ by a factor of three and we obtain different signs. This inverted sign is particularly inconvenient in the vorticity equation, where it results in an explicitly unstable term $\partial_{t} \omega=$ $\ldots+\mu_{i}^{n c} q^{2} / \varepsilon_{g}^{2} \omega$. There are several approaches to obtain the correct sign. One possibility is to use different closure relations for Ohm's law and the vorticity equation that are supposed to be equivalent, but result in different signs ${ }^{60,61}$.

Another approach is to split all fields $f$ into a resonant part $\bar{f}$ that follows the helicity of our resonant surface, and a nonresonant part $\tilde{f}$. Applying this to the parallel velocity equation (23), we find that equilibrium conditions require ${ }^{59}$ :

$$
\partial_{\theta}\left(\hat{\pi}_{\| i}+\hat{p}\right)=0
$$

with $\pi_{\| i}$ appropriately normalised. This information is lost when we perform the toroidal average, since then these two terms in the parallel momentum equation are zero. We can reinsert this information in our equation system by setting

$$
p_{i}+p_{e}=\bar{p}_{i}+\bar{p}_{e}-\hat{\pi}_{\| i}
$$

in the vorticity equation. This introduces another neoclassical term of the same form, so that we finally obtain:

$$
\partial_{t} \omega=\ldots-\mu_{i}^{n c}\left(\frac{q}{\varepsilon_{g}}\right)^{2}\left(\partial_{x} u_{\theta}+k_{i} \partial_{x} T_{i}\right)
$$

We recover the neoclassical term of (19) that we had obtained from the first closure relation. Nevertheless, we are still left with different neoclassical terms for the ion pressure equation. Because of these difficulties of obtaining a correct sign for the neoclassical friction term in the vorticity, we decided not to use the CGL-approach. Instead, we preferred the simpler closure on the viscous force that directly yields a correct sign. This closure is sufficient for our current slab geometry, even though it may not be appropriate once we decide to include the $\theta$-dependence of the equilibrium magnetic field explicitly into our model.

${ }^{1}$ M. Holzl, S. Gunter, and ASDEX Upgrade Team. Phys. Plasm., 15:072514,

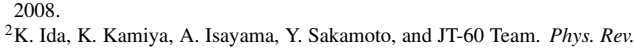
${ }^{2}$ K. Ida, K. Kamiya, A. Is
Lett., 109:065001, 2012.

${ }^{3}$ T. Estrada, E. Ascasibar, E. Blanco, A. Cappa, C. Hidalgo, K. Ida, A. Lopez-Fraguas, and B. Ph van Milligen. Nucl. Fusion, (56):026011, 2016.
${ }^{4}$ K. Ida, T. Kobayashi, M. Yoshinuma, Y. Susuki, Y. Narushima, T.E. Evans, S. Tsuchiya, S. Inagaki, and K. Itoh. Nucle ar Fusion, 56:092001, 2016. ${ }_{5}^{5}$ L. Bardoczi, T. L. Rhodes, T. A. Carter, A. Banñon Navarro, W. A. Peebles, F. Jenko, and G. McKee. Phys. Rev. Lett., 116:215001, 2016.

${ }^{6}$ L. Bardoczi, T. L. Rhodes, A. Banon Navarro, C. Sung, T. A. Carter, R. J. La Haye, G. R. McKee, C. C. Petty, C. Chrystal, and F. Jenko. Multi-field/scale interactions of turbulence with neoclassical tearing mode magnetic islands in the DIII-D tokamak. Physics of Plasmas, 24(5):056106, 2017. ISSN 10897674. doi:10.1063/1.4977533.

${ }^{7}$ L. Bardoczi, T. A. Carter, R. J. La Haye, T. L. Rhodes, and G. R. McKee. Phys. Plasm., (24):122503, 2017.

${ }^{8}$ K. J. Zhao, Y. Nagashima, F. M. Li, Yuejiang Shi, P. H. Diamond, J. Q. Dong, K. Itoh, S.-I. Itoh, G. Zhuang, H. Liu, et al. Nucl. Fusion, (57): 126006, 2017.

${ }^{9}$ K. Ida, T. Kobayashi, M. Ono, G. R. McKee, and M. E. Austin. Phys. Rev. Lett., 120:245001, 2018.

${ }^{10}$ P. J. Sun, Y. D. li, X. D. Zhang, G. J. Wu, L. Q. Xu, R. Chen, Q. Li, H. L. Zhao, J. Z. Zhang, T. H. Shi, Y. M. Wang, B. Lyu, L. Q. Hu, J. Li, and EAST the Team. Nucl. Fusion, 58:016003, 2018.

${ }^{11}$ A. Ishizawa and N. Nakajima. Phys. Plasmas, 17:072308, 2010.

${ }^{12}$ M. Muraglia, O. Agullo, S. Benkadda, M. Yagi, X. Garbet, and A. Sen. Phys. Rev. Lett., 107:095003, 2011.

${ }^{13}$ A. Ishizawa and F. L. Waelbroeck. Phys. Plasm., 20:122301, 2013.

${ }^{14}$ O. Agullo, M. Muraglia, A. Poyé, S. Benkadda, M. Yagi, X. Garbet, and A. Sen. A signature for turbulence driven magnetic islands. Phys. Plasma, 21:092303, 2014

${ }^{15} \mathrm{~J}$. Li, Y. Kishimoto, and Z. X. Wangt. Phys. Plasm., 21:020703, 2014.

${ }^{16}$ A. Poyé, O. Agullo, M. Muraglia, X. Garbet, S. Benkadda, A. Sen, and N. Dubuit. Phys. Plasma, 22:030704, 2015.

${ }^{17}$ W A Hornsby, P Migliano, R Buchholz, D Zarzoso, F J Casson, E Poli, and A G Peeters. On seed island generation and the non-linear self-consistent interaction of the tearing mode With electromagnetic gyro-kinetic turbulence. Plasma Physics and Controlled Fusion, 57(5):054018, 2015. URL http: //stacks . iop.org/0741-3335/57/i=5/a=054018.

${ }^{18}$ O. Agullo, M. Muraglia, S. Benkadda, A. Poyé, N. Dubuit, X. Garbet, and A. Sen. Nonlinear dynamics of turbulence driven magnetic islands. I. Theoretical aspects. Physics of Plasmas, 24(4):042308, 2017. doi: 10.1063/1.4981229. URL https://doi.org/10.1063/1.4981229.

${ }^{19}$ O. Agullo, M. Muraglia, S. Benkadda, A. Poyé, N. Dubuit, X. Garbet, and A. Sen. Nonlinear dynamics of turbulence driven magnetic islands. II. Numerical simulations. Physics of Plasmas, 24(4):042309, 2017. doi: 10.1063/1.4981230. URL https://doi.org/10.1063/1.4981230.

${ }^{20}$ M. Muraglia, O. Agullo, A. Poyé, S. Benkadda, N. Dubuit, X. Garbet, and A. Sen. Amplification of a turbulence driven seed magnetic island by bootstrap current. Nuclear Fusion, 57(7):072010, may 2017. doi:10.1088/1741-4326/aa654d. URL https://doi.org/10. $1088 \% 2 \mathrm{~F} 1741-4326 \% 2 \mathrm{Faa} 654 \mathrm{~d}$

${ }^{21}$ Jae Min Kwon, S. Ku, M. J. Choi, C. S. Chang, R. Hager, E. S. Yoon, H. H. Lee, and H. S. Kim. Gyrokinetic simulation study of magnetic island effects on neoclassical physics and micro-instabilities in a realistic KSTAR plasma. Physics of Plasmas, 25(5):052506, 2018. ISSN 10897674. doi: 10.1063/1.5027622.

${ }^{22}$ Huishan Cai. A mechanism of neoclassical tearing modes onset by drift wave turbulence. Nuclear Fusion, 59:026009, 2019

${ }^{23}$ A Ishizawa, Y Kishimoto, and Y Nakamura. Multi-scale interactions between turbulence and magnetic islands and parity mixture-a review. Plasma Physics and Controlled Fusion, 61(5):054006, mar review. doi:10.1088/1361-6587/ab06a8. URL https://doi.org/10. 2019. doi: $10.1088 / 1361-6587 /$ a
1088\%2F $1361-6587 \% 2 \mathrm{Fab} 06 \mathrm{a} 8$

$1088 \% 2 F 1361-6587 \% 2 F a b 06 a 8$.
${ }^{24}$ M. Muraglia, O. Agullo, S. Benkadda, M. Yagi, X. Garbet, and A. Sen. Generation and amplification of magnetic islands by drift interchange turbulence. Physical Review Letters, 107(9):1-5, 2011. ISSN 00319007. doi: 10.1103/PhysRevLett.107.095003.

${ }^{25}$ W. A. Hornsby, P. Migliano, R. Buchholz, D. Zarzoso, F. J. Casson, E. Poli, and A. G. Peeters. On seed island generation and the non-linear selfconsistent interaction of the tearing mode with electromagnetic gyro-kinetic turbulence. Plasma Physics and Controlled Fusion, 57(5), 2015.

${ }^{26}$ S. P. Gerhardt, D P Brennan, J. Buttery, R. J. La Haye, S. Sabbagh, E. Strait, M. Bell, R. Bell, E. Fredrickson, and D. Gates. Relationship between onset thresholds, trigger types and rotation shear for the $\mathrm{m} / \mathrm{n}=2 / 1$ neoclassical 
tearing mode in a high- $\backslash$ beta spherical torus. Nuclear Fusion, 49(3), 2009. ${ }^{27}$ P. Hill, F. Hariri, and M. Ottaviani. The effect of magnetic islands on Io Temperature Gradient turbulence driven transport. Physics of Plasmas, 22 (4), 2015. ISSN 10897674. doi:10.1063/1.4919031.

${ }^{28}$ Olivier Izacard, Christopher Holland, Spencer D. James, and Dylan P. Brennan. Dynamics of ion temperature gradient turbulence and transport with static magnetic island. Physics of Plasmas, 23(2), 2016. ISSN 10897674 doi:10.1063/1.4941704.

${ }^{29}$ Z. X. Wang, J. Q. Li, Y. Kishimoto, and J. Q. Dong. Magnetic-islandinduced ion temperature gradient mode. Physics of Plasmas, 16(6):1-5, 2009. ISSN 1070664X. doi:10.1063/1.3166600.

${ }^{30}$ T.C. Hender, P Buratti, F.J. Casson, B Alper, Yu. F Baranov, M Baruzzo, C.D. Challis, F Koechl, K.D. Lawson, C Marchetto, M.F.F. Nave, T. Pütterich, and S. Reyes Cortes. The role of MHD in causing impurity peaking in JET hybrid plasmas. Nuclear Fusion, 56(6) 066002, 2016. ISSN 0029-5515. doi:10.1088/0029-5515/56/6/066002 URL http://stacks.iop.org/0029-5515/56/i=6/a=066002?key= crossref. $5 \mathrm{f} 6 \mathrm{a} 8 \mathrm{f} 0 \mathrm{f} 1 \mathrm{f} 43 \mathrm{bf} 86 \mathrm{e} 1670 \mathrm{bdc} 08 \mathrm{~d} 106 \mathrm{dd}$.

${ }^{31}$ M. Sertoli, C. Angioni, and T. Odstrcil. Parametric dependencies of the experimental tungsten transport coefficients in ICRH and ECRH assisted ASDEX Upgrade H-modes. Physics of Plasmas, 24(11), 2017. ISSN 10897674. doi: $10.1063 / 1.4996412$

${ }^{32}$ S. I. Braginskii. Transport processes in a plasma, 1965. URL http://people.hao.ucar.edu/judge/homepage/PHSX515/ fall2012/Braginskii1965.pdf.

${ }^{33}$ A. I. Smolyakov. Gyroviscous forces in a collisionless plasma with temperature gradients. Canadian Journal of Physics, 76(4):321-331, 1998. ISSN 00084204 doi:10.1139/cjp-76-4-321.

${ }^{34}$ T. A. Gianakon, S. E. Kruger, and C. C. Hegna. Heuristic closures for numerical simulations of neoclassical tearing modes. Physics of Plasmas, 9(2):536, 2002. ISSN 1070664X. doi:10.1063/1.1424924.

${ }^{35}$ J. D. Callen and K. C. Shaing. A pressure-gradient-driven tokamak "resistive magnetohydrodynamic" instability in the banana-plateau collisionality regime. Physics of Fluids, 28(6):1845-1858, 1985. ISSN 10706631. doi: $10.1063 / 1.864928$.

${ }^{36}$ S. P. Hirshman and D. J. Sigmar. Neoclassical Transport of Impurities in Tokamak Plasmas. Nuclear Fusion, 21(9):1079-1201, 1981.

${ }^{37}$ M. Yagi, S. Yoshida, S. I. Itoh, H. Naitou, H. Nagahara, J. N. Leboeuf, K. Itoh, T. Matsumoto, S. Tokuda, and M. Azumi. Nonlinear simulation of tearing mode based on 4-field RMHD model. Nuclear Fusion, 45(8): 900-906, 2005. ISSN 00295515. doi:10.1088/0029-5515/45/8/018.

${ }^{38}$ Atsushi Furuya, Masatoshi Yagi, and Sanae I. Itoh. Linear analysis of neoclassical tearing mode based on the four-field reduced neoclassical MHD equation. Journal of the Physical Society of Japan, 72(2):313-319, 2003. ISSN 00319015. doi:10.1143/JPSJ.72.313.

${ }^{39}$ F. Orain, M. Bécoulet, G. Dif-Pradalier, G. Huijsmans, S. Pamela, E. Nardon, C. Passeron, G. Latu, V. Grandgirard, A. Fil, A. Ratnani, I. Chapman, A. Kirk, A. Thornton, M. Hoelzl, and P. Cahyna. Non-linear magnetohydrodynamic modeling of plasma response to resonant magnetic perturbations. Physics of Plasmas, 20(10), 2013. ISSN 1070664X. doi turbations. Physics

${ }^{40}$ The Boltzmann equation and the one-fluid hydromagnetic equations in the absence of particle collisions. Proceedings of the Royal Society of London Series A. Mathematical and Physical Sciences, pages 112-118, 1956.

${ }^{41}$ R. D. Hazeltine, M. Kotschenreuther, and P. J. Morrison. A four-field mode for tokamak plasma dynamics. Physics of Fluids, 28(8):2466-2477, 1985.

${ }^{42}$ M. Yagi, T. Ueda, S. I. Itoh, M. Azumi, K. Itoh, P. H. Diamond, and T. S. Hahm. Turbulence spreading in reversed shear plasmas. Plasma Physic and Controlled Fusion, 48(5 A), 2006. ISSN 07413335. doi:10.1088/07413335/48/5A/S42

${ }^{43}$ M. Yagi, J. P. Wang, Y. B. Kim, and M. Azumi. Ion-temperature-gradientdriven modes in neoclassical regime. Physics of Fluids B, 5(4):1179-1187, 1993. ISSN 08998221. doi:10.1063/1.860908.

${ }^{44}$ M. Muraglia, O. Agullo, S. Benkadda, X. Garbet, P. Beyer, and A. Sen.
Nonlinear dynamics of magnetic islands imbedded in small-scale turbulence. Physical Review Letters, 103(14):2-5, 2009. ISSN 00319007. doi: 10.1103/PhysRevLett.103.145001.

${ }^{45}$ H. Nordman, J. Weiland, and A. Jarmén. Simulation of toroidal drift mode turbulence driven by temperature gradients and electron trapping. Nuclear Fusion, 30(6):983-996, jun 1990. doi:10.1088/0029-5515/30/6/001. URL https://doi .org/10.1088\%2F0029-5515\%2F30\%2F6\%2F001.

${ }^{46}$ A. I. Smolyakov, P. H. Diamond, and M. V. Medvedev. Role of ion diamagnetic effects in the generation of large scale flows in toroidal ion temperature gradient mode turbulence. Physics of Plasmas, 7(10):3987-3992, 2000. ISSN 1070664X doi:10.1063/1.1289514.

${ }^{47}$ A. Jarmén, P. Andersson, and J. Weiland. Fully toroidal ion temperature gradient driven drift modes. Nuclear Fusion, 27(6):941-949, jun 1987. doi:10.1088/0029-5515/27/6/006. URL https://doi.org/10. $1088 \% 2 \mathrm{~F} 0029-5515 \% 2 \mathrm{~F} 27 \% 2 \mathrm{~F} 6 \% 2 \mathrm{~F} 006$

${ }^{48}$ A. M. Dimits, G. Bateman, M. A. Beer, B. I. Cohen, W. Dorland, G. W. Hammett, C. Kim, J. E. Kinsey, M. Kotschenreuther, A. H. Kritz, L. L. Lao, J. Mandrekas, W. M. Nevins, S. E. Parker, A. J. Redd, D. E. Shumaker, R. Sydora, and J. Weiland. Comparisons and physics basis of tokamak transport models and turbulence simulations. Phys.Plasmas, 7(3):969-983, 2000.

${ }^{49}$ E. G. Harris. On a Plasma Sheath Separating Regions of Oppositely Directed Magnetic Field. Il nuovo cimento, 23(1), 1962.

${ }^{50}$ Masatoshi Yagi, Masahiro Wakatani, Hideo Sugama, B. G. Hong, and W. HORTON. Ion-temperature-gradient-driven drift mode coupled to the resistive interchange mode in a heliotron/torsatron. Journal of the Physical Society of Japan, 58(12):4265-4268, 1989. doi:10.1143/JPSJ.58.4265. URL https: //doi.org/10.1143/JPSJ .58.4265.

${ }^{51}$ B.-G. Hong, W. Horton, S. Hamaguchi, M. Wakatani, M. Yagi, and H. Sugama. Transition from resistive-g to i- driven turbulence in stellarator systems. Physics of Fluids B: Plasma Physics, 3(7):1638-1643, 1991 doi:10.1063/1.859683. URL https://doi.org/10.1063/1.859683.

${ }^{52} \mathrm{~W}$. Horton. Drift waves and transport. Rev. Mod. Phys., 71:735-778, Apr 1999. doi:10.1103/RevModPhys.71.735. URL https://link . aps . org/ doi/10.1103/RevModPhys.71.735.

${ }^{53}$ J. Y. Kim, Y. Kishimoto, M. Wakatani, and T. Tajima. Poloidal shear flow effect on toroidal ion temperature gradient mode: A theory and simulation. Physics of Plasmas, 3(10):3689-3695, 1996. ISSN 1070664X. doi: $10.1063 / 1.871939$

${ }^{54}$ G. Rewoldt, M. A. Beer, M. S. Chance, T. S. Hahm, Z. Lin, and W. M. Tang. Sheared rotation effects on kinetic stability in enhanced confinement tokamak plasmas, and nonlinear dynamics of fluctuations and flows in axisymmetric plasmas. Physics of Plasmas, 5(5):1815-1821, 1998. ISSN 1070-664X. doi:10.1063/1.872851.

${ }^{55} \mathrm{P}$ W Terry. Suppression of turbulence and transport by sheared flow. Reviews of Modern Physics, 72(1):109-165, 2000.

${ }^{56} \mathrm{~S}$. Hamaguchi and W. Horton. Effects of sheared flows on ion-temperaturegradient-driven turbulent transport. Physics of Fluids B: Plasma Physics, 4 (2):319-328, 1992. doi:10.1063/1.860280. URL https://doi.org/10. $1063 / 1.860280$

${ }^{57} \mathrm{P}$. Beyer. Turbulence et transport dans les plasmas chauds magnétisés. $\mathrm{PhD}$ thesis, Aix-Marseille University, 2004.

${ }^{58} \mathrm{P}$. Helander and D. J. Sigmar. Collisional Transport in Magnetized Plasmas. Cambridge University Press, 2002

${ }^{59}$ A. I. Smolyakov and E. Lazzaro. On neoclassical effects in the theory of magnetic islands. Physics of Plasmas, 11(9):4353-4360, 2004. ISSN 1070664X doi:10.1063/1.1775007.

${ }^{60}$ J. D. Callen. Viscous Forces Due To Collisional Parallel Stresses For Extended MHD Codes. (UW-CPTC 09-6R):1-30, 2010. doi 10.1063/1.3335486. URL http://homepages.cae.wisc.edu/ $\left\{\sim\right.$ callen/UW-CPTC $\left\{\_\right\} 09-6 R$.pdf.

${ }^{61}$ Patrick Maget, Olivier Février, Xavier Garbet, Hinrich Lütjens, JeanFrancois Luciani, and Alain Marx. Extended magneto-hydro-dynamic model for neoclassical tearing mode computations. Nuclear Fusion, 56 (8):086004, 2016. ISSN 17414326. doi:10.1088/0029-5515/56/8/086004. 


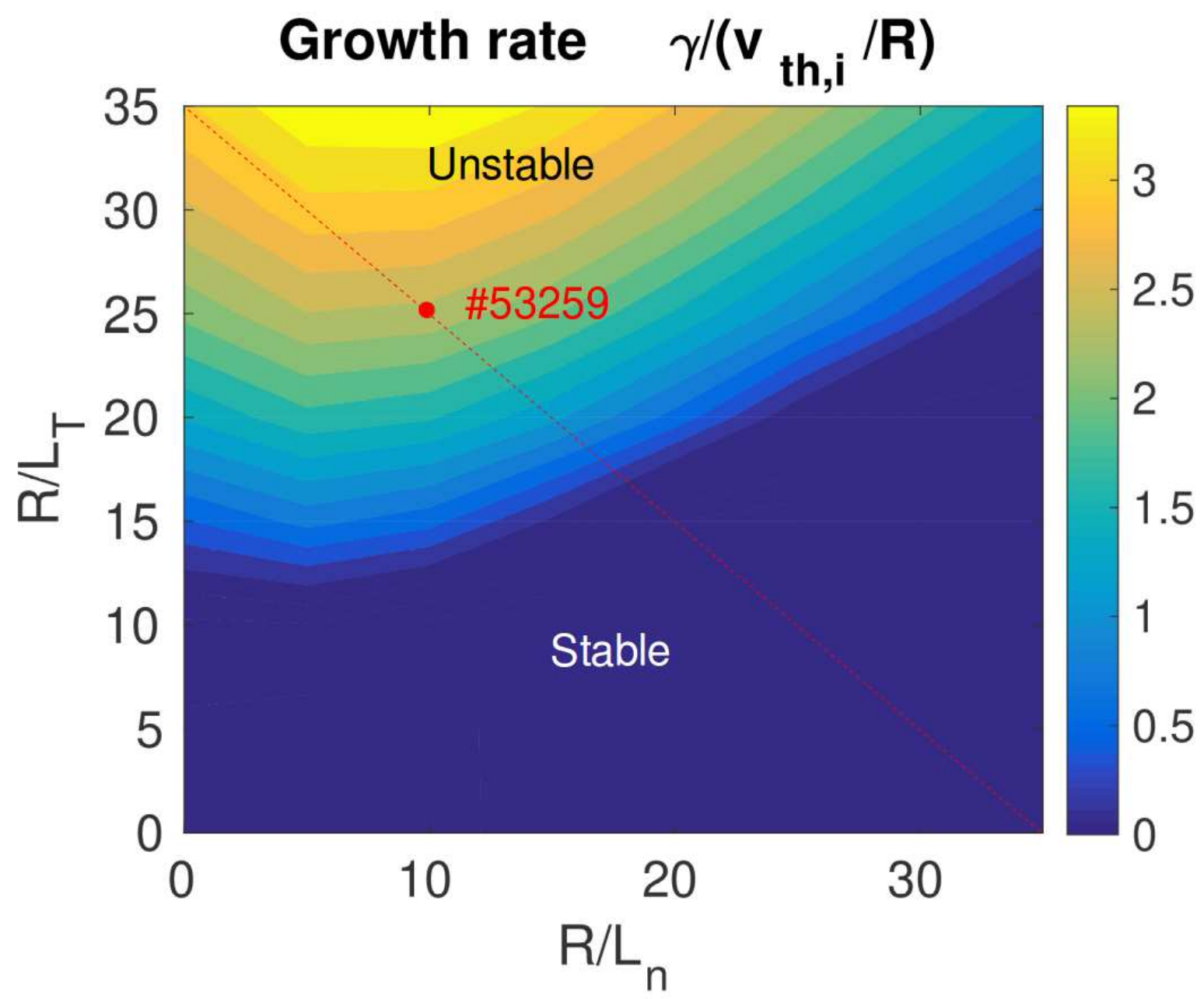




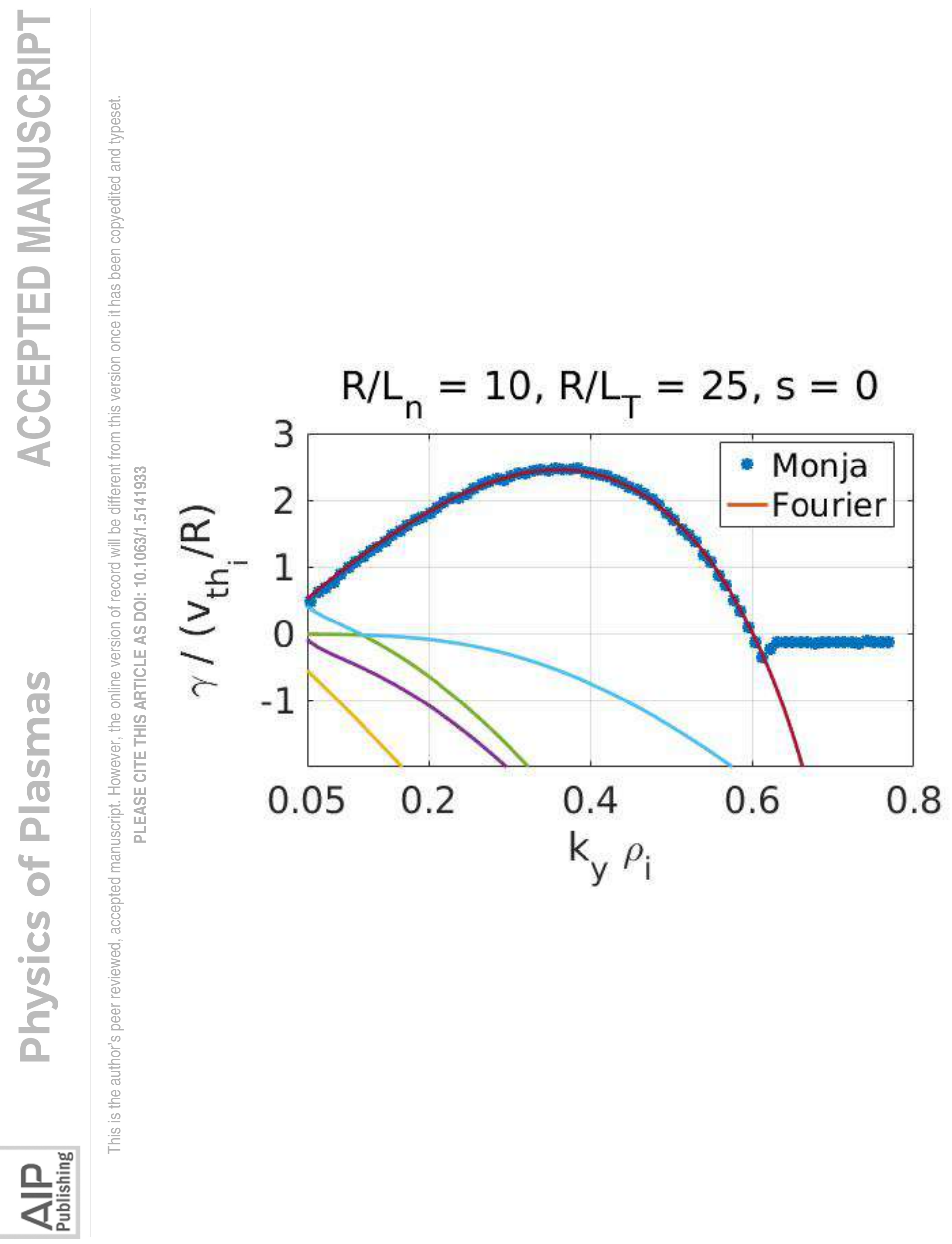




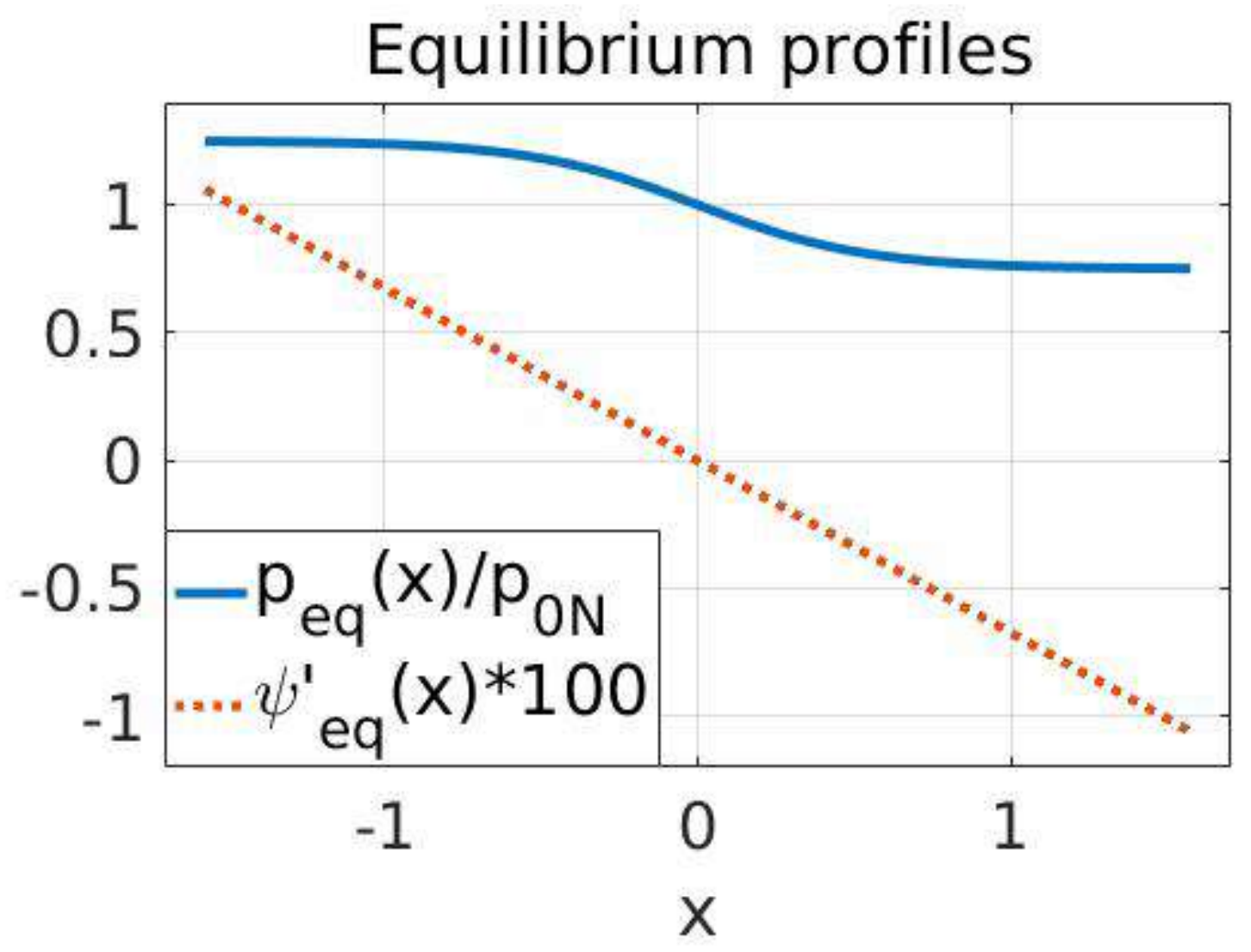

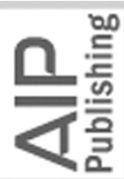




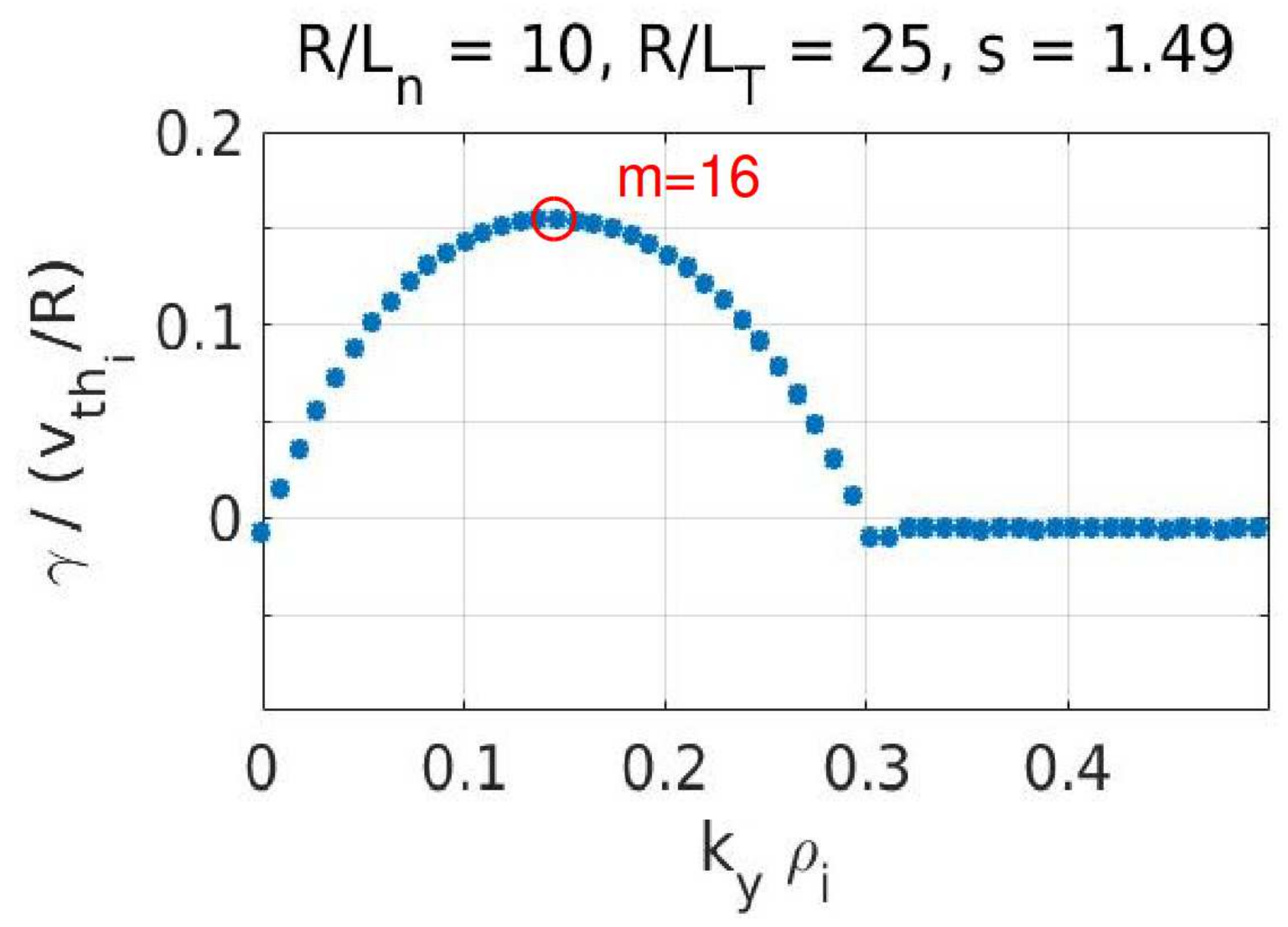

은 


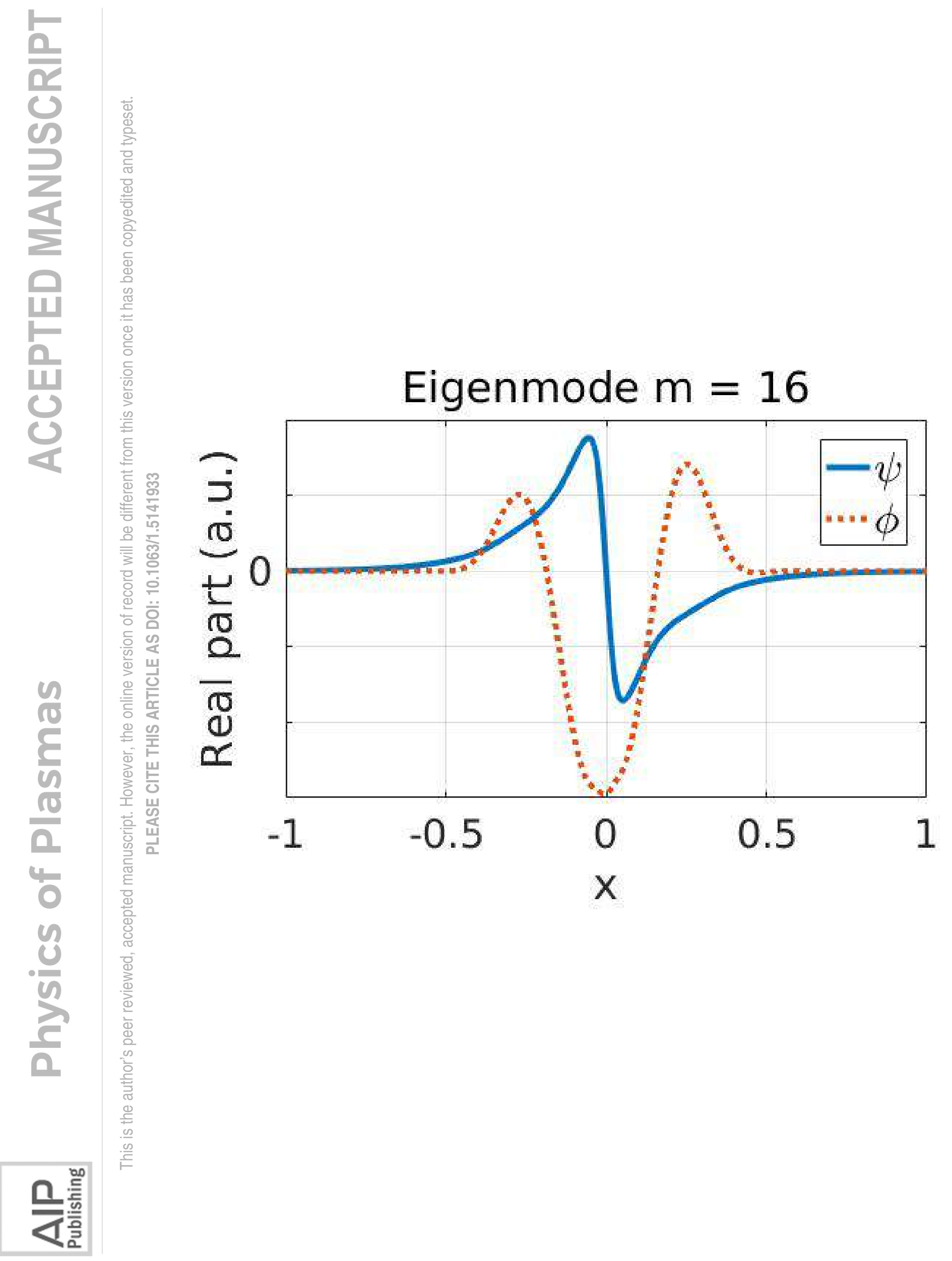




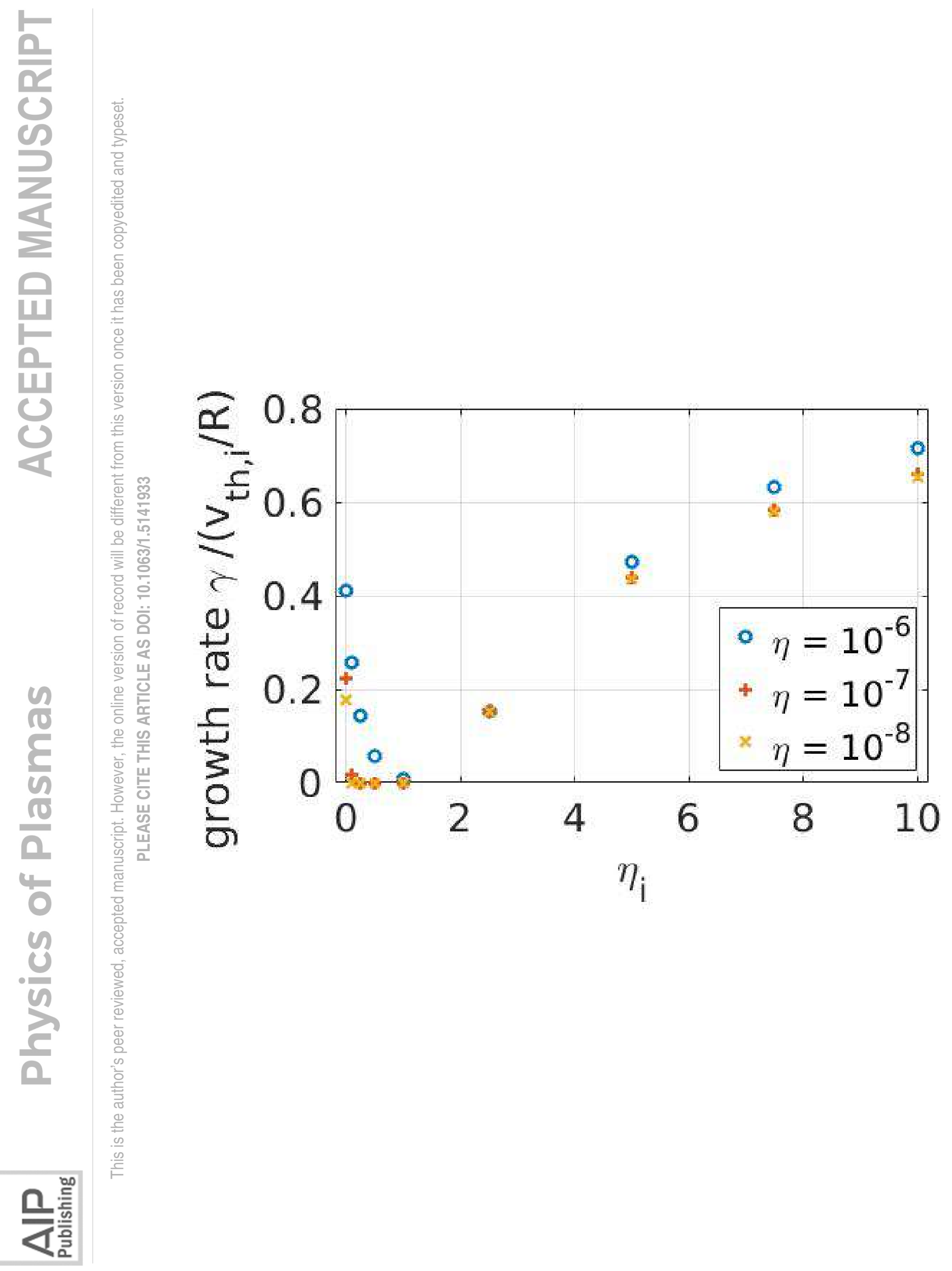




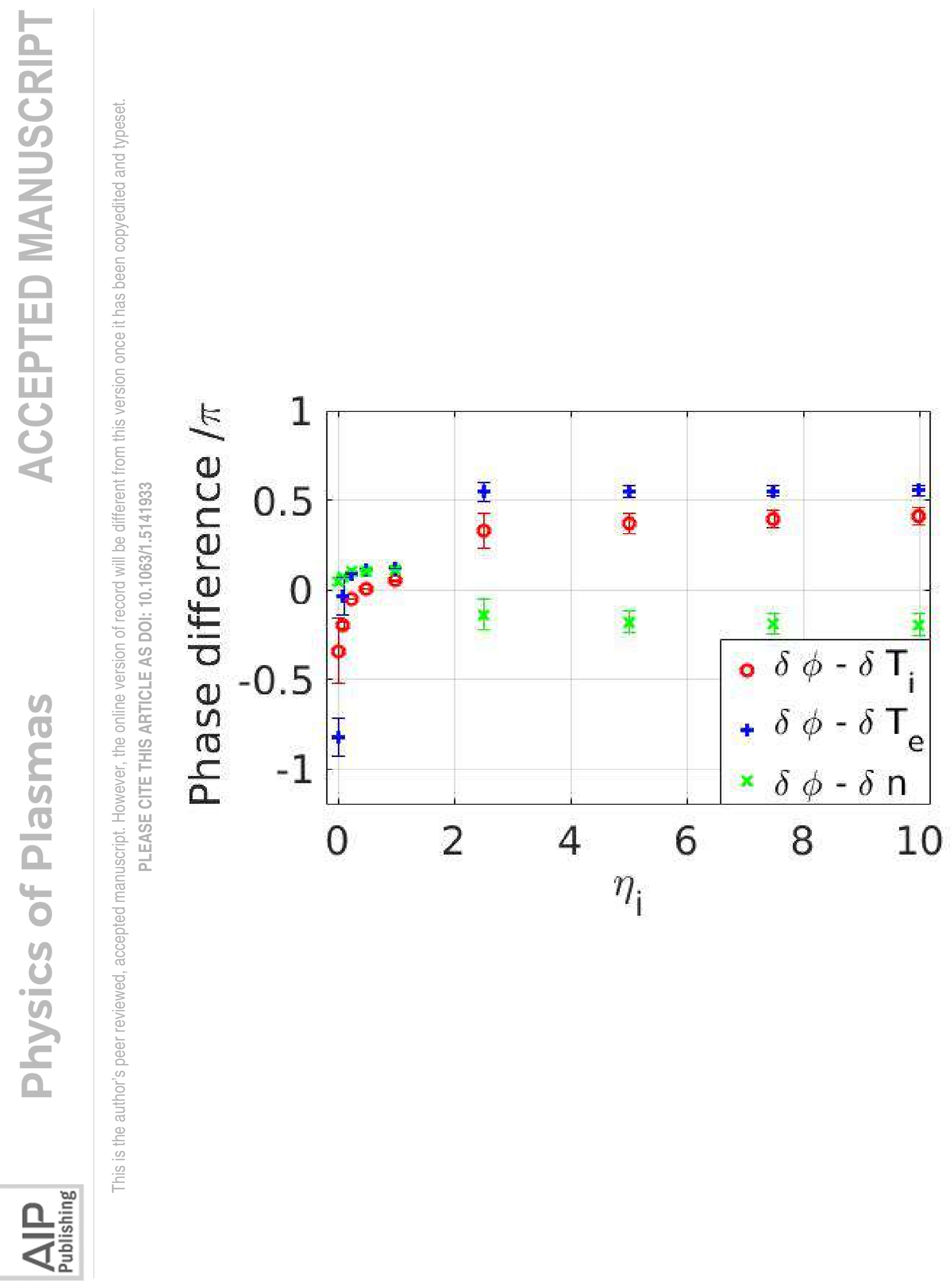



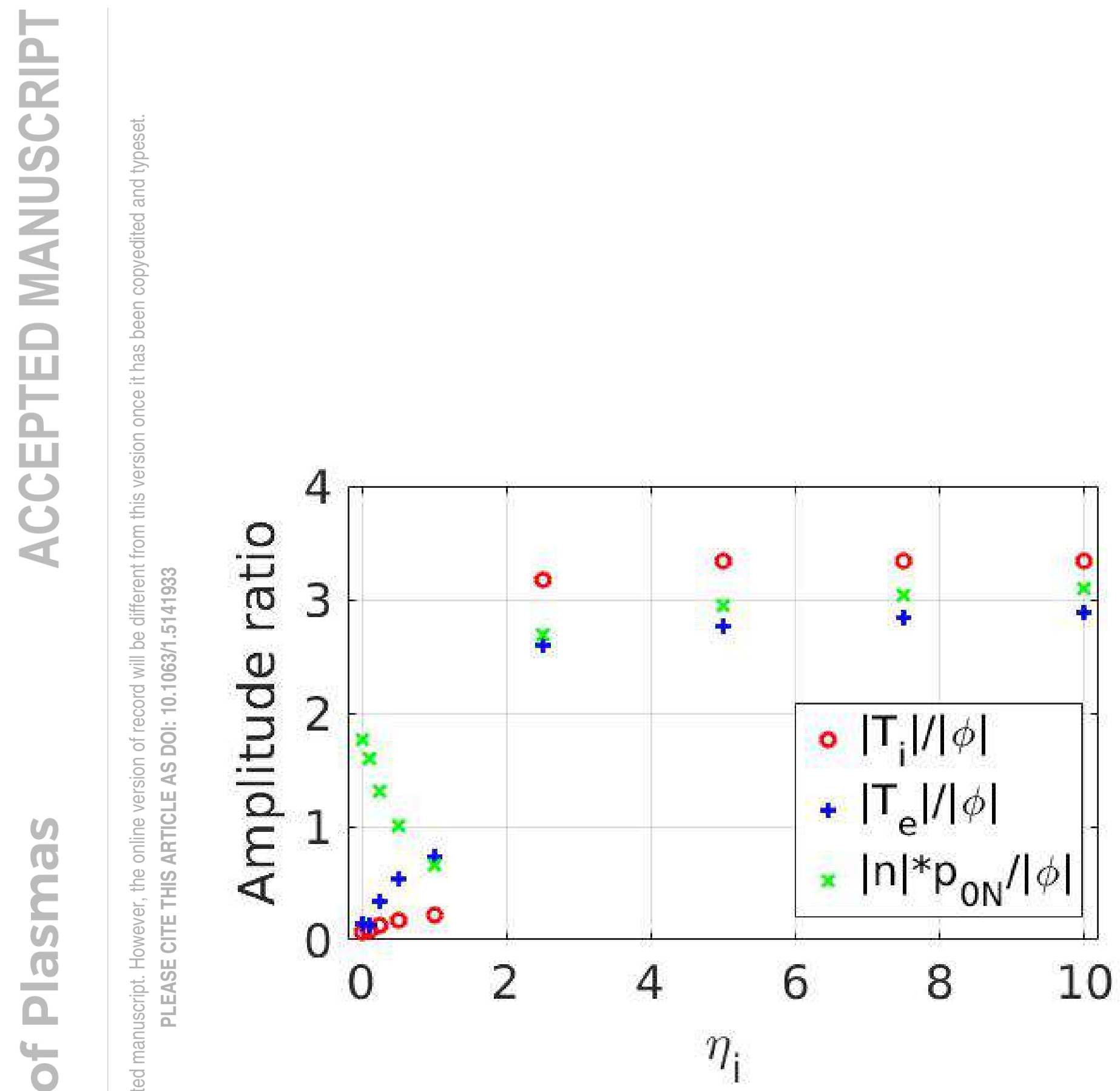

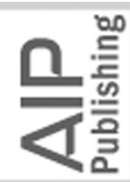




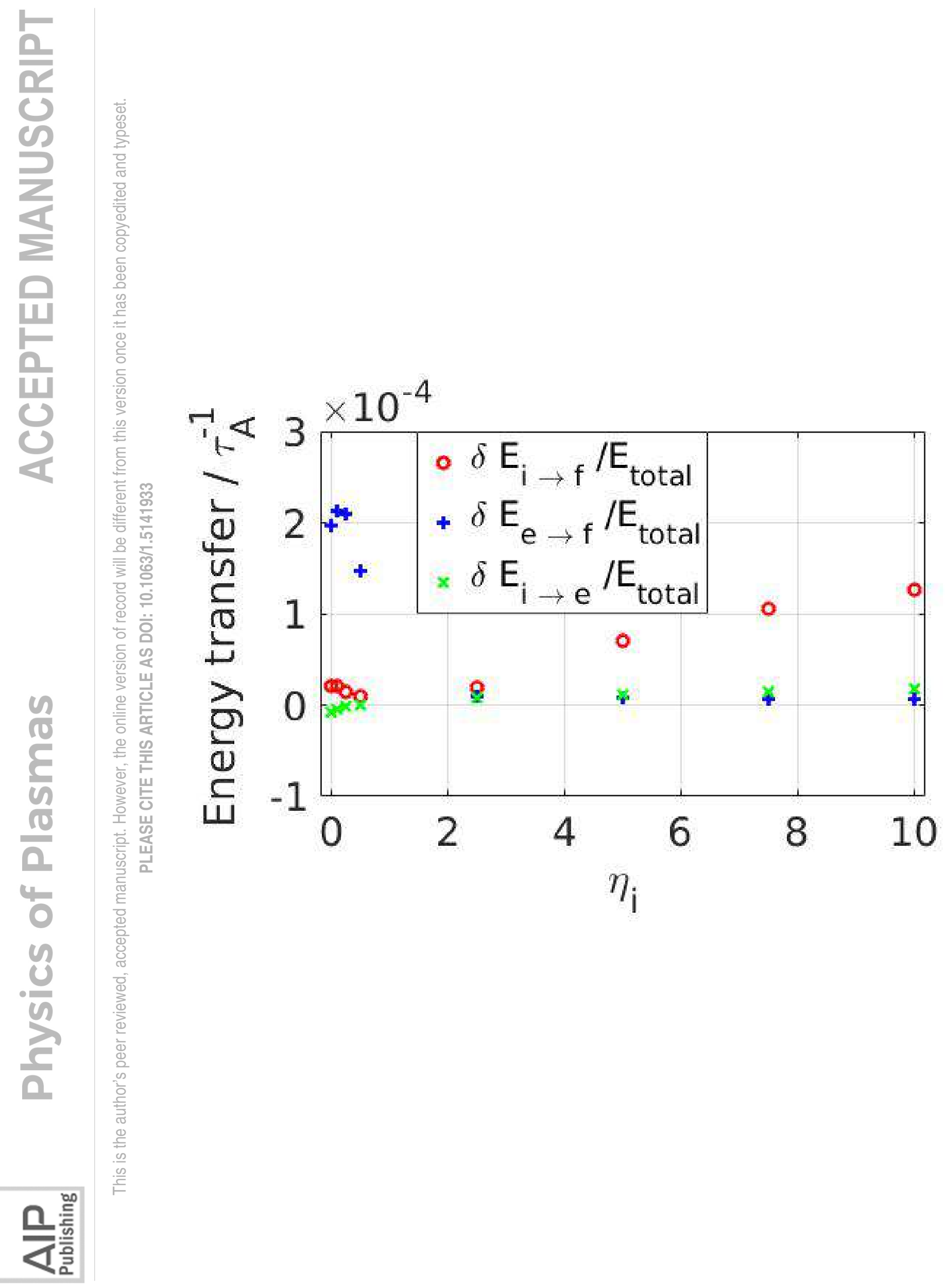




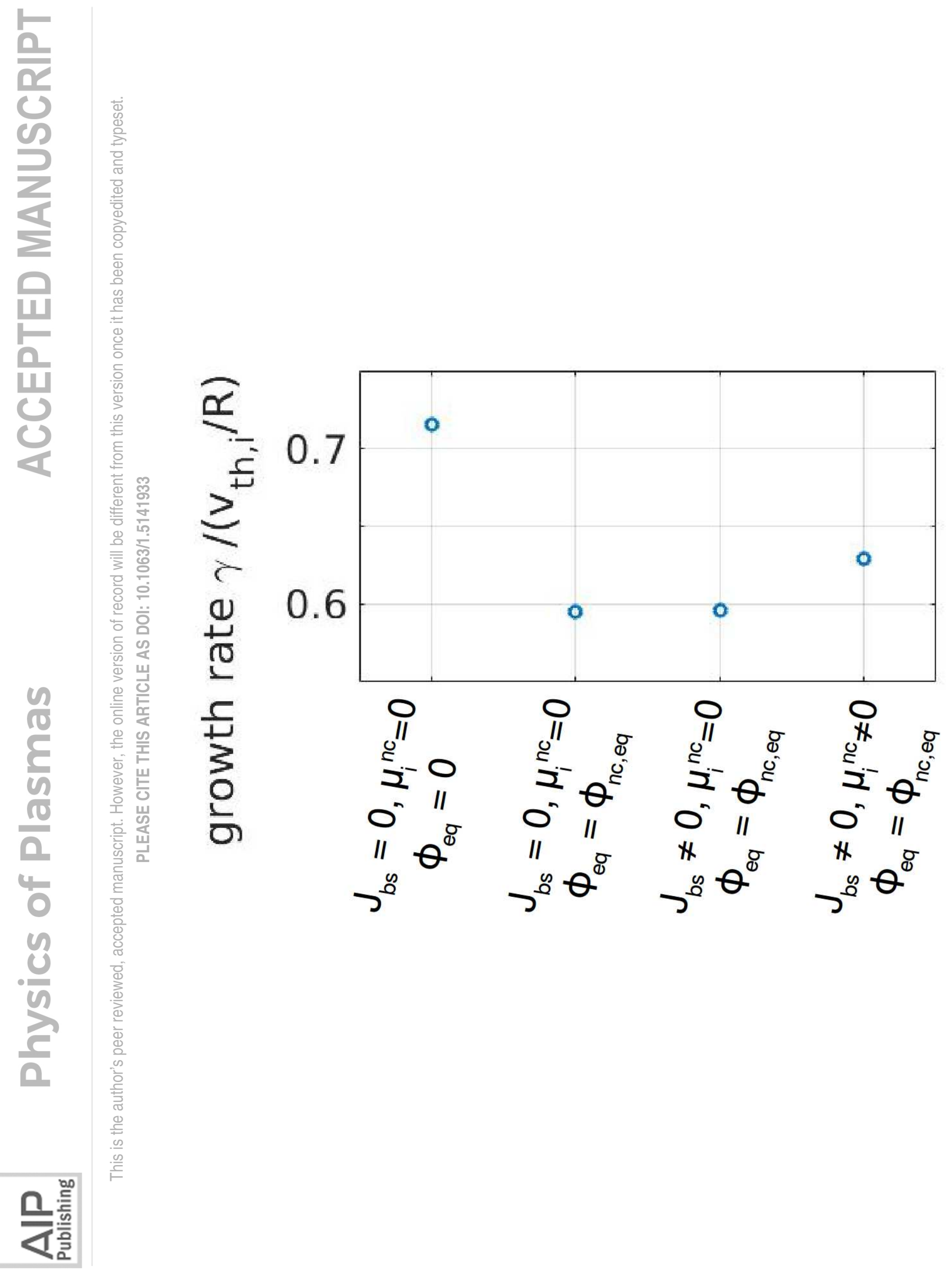




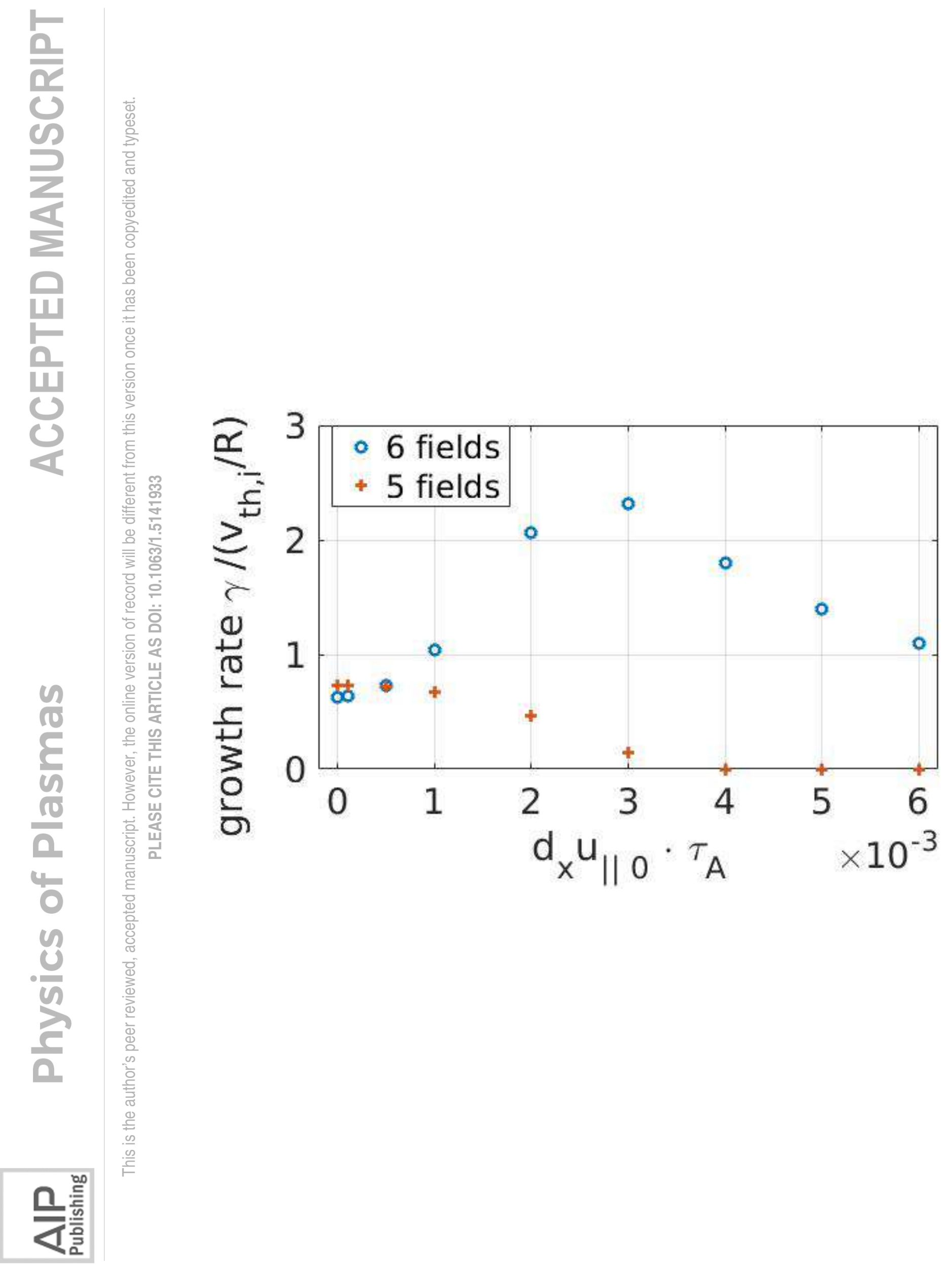




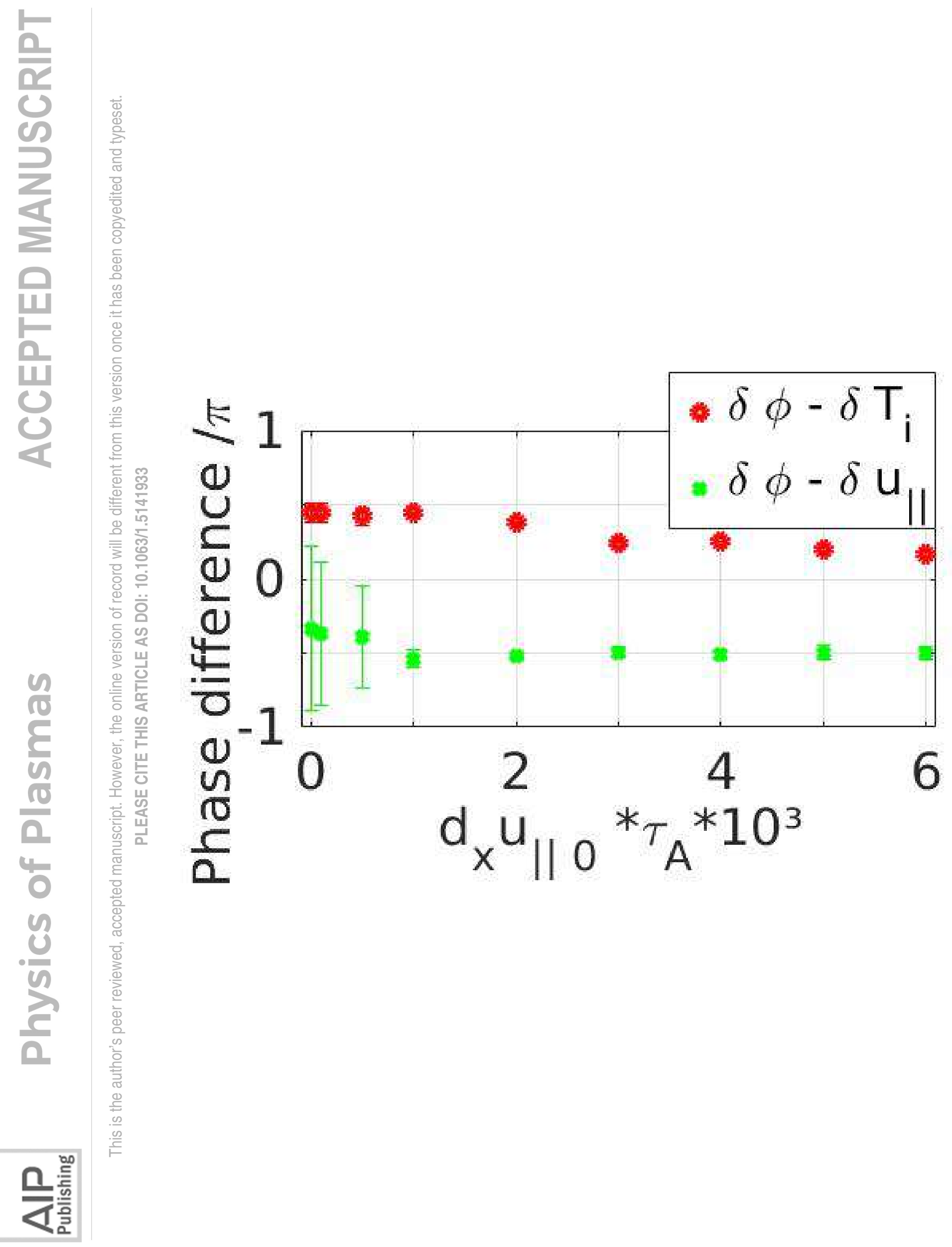




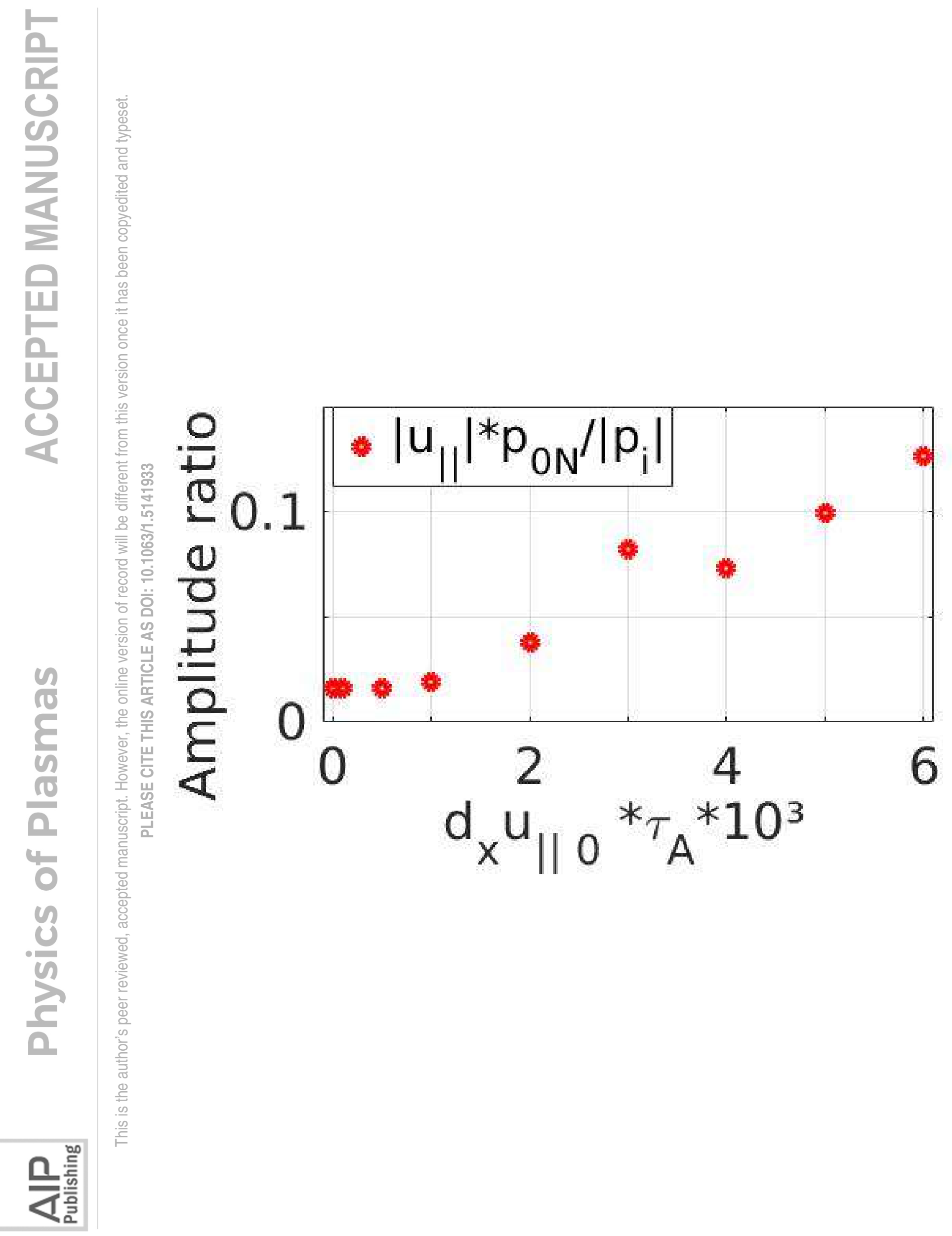




\section{$\phi$ fluctuations (a.u.) $d_{x} u_{\| 0}=10^{-4} \tau_{A}^{-1}$}

15

10

$>$

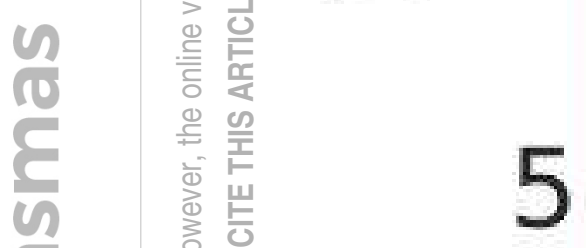

0

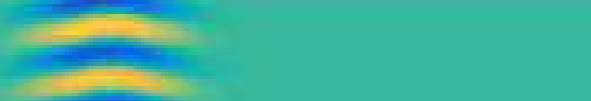

tons

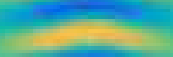

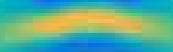

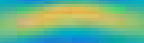

$=1$

19

(a)

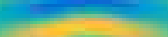

(25)

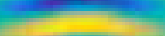

(a)

(2)

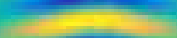

(2)

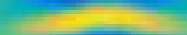

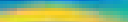

$-1$

0

1

$\mathrm{X}$

踯衰 
$\phi$ fluctuations (a.u.) $d_{x} u_{\| 0}=3 \cdot 10^{-3} \tau_{A}{ }^{-1}$

㞺

15

10

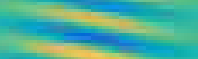

$\operatorname{sinsm}$

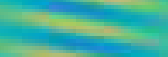

masens

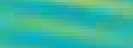

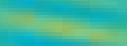

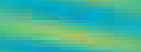

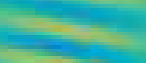

$>$

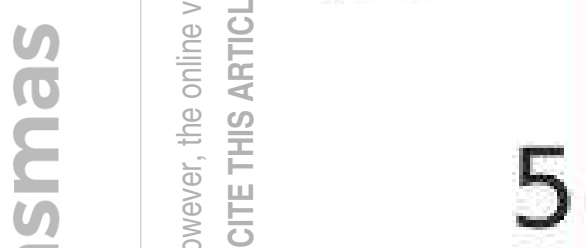

$\frac{\pi}{2}$

는

은 땐

뭉

0

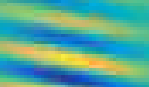

sises:

20

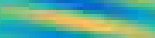

$\sqrt{20}$

$\sin$

17mas:

$\sin$

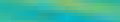

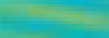

$-\sqrt{\sin }$

$\sin$

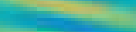

tis

$\sqrt{305}$

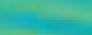

$\sin 20$

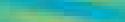

10:

$\sin (\sin 20$

30

$-1$

0

1

$\mathrm{X}$

는 